NATIONAL AERONAUTICS AND SPACE ADMINISTRATION

Technical Report 32-1525

\title{
Finite Element Appendage Equations for Hybrid Coordinate Dynamic Analysis
}

Peter W. Likins
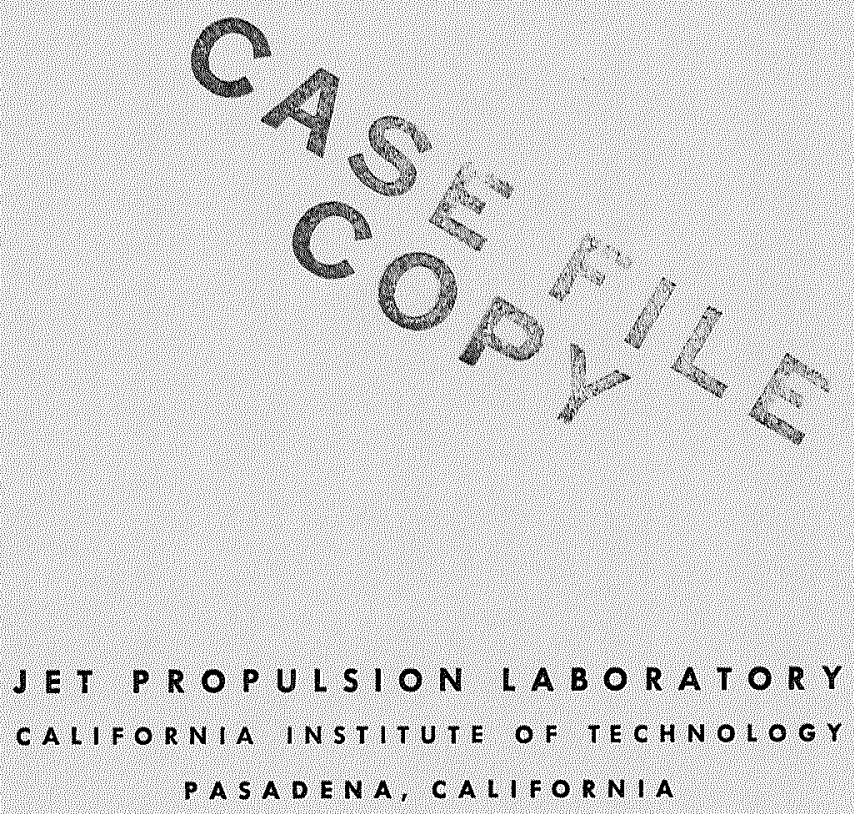
Technical Report 32-1525

\title{
Finite Element Appendage Equations for Hybrid Coordinate Dynamic Analysis
}

\author{
Peter W. Likins
}

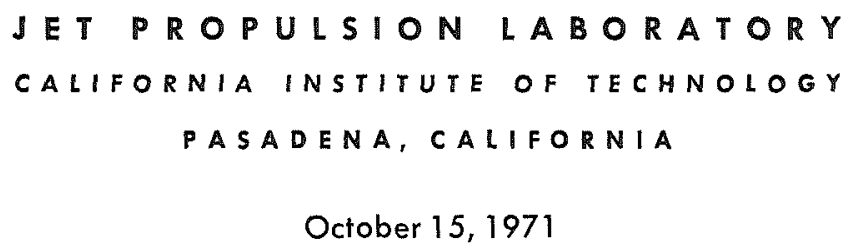


Prepared Under Contract No. NAS 7-100

National Aeronautics and Space Administration 


\section{Preface}

The work described in this report was performed under the cognizance of the Guidance and Control Division of the Jet Propulsion Laboratory, supported by NASA Contracts NAS 7-100 and NAS 8-26214. The author, an associate professor at UCLA, is a consultant to JPL. 


\section{Acknowledgment}

The procedures described in this report were developed in preliminary form under JPL auspices. As Principal Investigator of a UCLA research contract with NASA Manned Space Flight Center (Contract NAS 8-26214) the author had the opportunity to apply these procedures to a proposed spinning spacecraft, Skylab $B$. The stimulus of this application and the interaction with others associated with this contract had significant impact on this report, which was substantially revised as a result of this experience. Thus the research and development program culminating in this report was, in effect, jointly sponsored by JPL and NASA MSFC. This study benefited from the technical and/or managerial support of Mr. H. K. Bouvier, Dr. E. L. Marsh, Dr. E. O. Weiner, and Dr. K. K. Gupta of JPL, Dr. F. J. Barbera of UCLA and North American Rockwell, Dr. S. M. Seltzer and Mr. D. Justice of MSFC, Dr. J. Patel of Brown Engineering, Dr. W. W. Hooker of Lockheed Palo Alto Research Laboratories, and Mr. A. S. Hopkins of UCLA and McDonnell-Douglas Corp. 


\section{Contents}

I. Introduction ..................... . . . 1

II. Appendage Idealization . . . . . . . . . . . . . . . . . . . 2

III. Finite Element Equations of Motion . . . . . . . . . . . . . . . 3

IV. Nodal Body Equations of Motion . . . . . . . . . . . . . . 11

V. Coordinate Transformations . . . . . . . . . . . 13

Vl. Perspective . . . . . . . . . . . . . . . . 18

Appendix. Proof of Eq. (99) . . . . . . . . . . . . . . . . . . . . . 19

Nomenclature . . . . . . . . . . . . . . . . 21

References . . . . . . . . . . . . . . . . . 25

Figure

1. Appendage idealization. . . . . . . . . . . . . . . . 2 


\section{Author's Note}

This report is intimately related to a previous publication, Dynamics and Control of Flexible Space Vehicles, Jet Propulsion Laboratory Technical Report 32-1329, Rev. 1, dated January 15, 1970, by the same author. The earlier report is much wider in scope, dealing with discrete coordinate methods and vehicle normal-mode coordinate methods as well as hybrid coordinate methods. Within the framework of the hybrid coordinate approach, the earlier report offers a small section (15 pages) on the equations of motion of a flexible appendage, and presents as well an extensive treatment of other ingredients of a hybrid coordinate formulation for a complete spacecraft.

The present report consists of a substantial expansion of the original section on the equations of motion of a flexible appendage, generalizing the appendage mathematical model to permit the distribution of mass throughout the finite elastic elements interconnecting the nodes at which all mass is concentrated in the earlier report. In addition, the present report provides a procedure for coordinate transformation which may be more efficient computationally than those considered in the earlier report. The present report is, however, much narrower in scope than its predecessor, so that it is best appreciated in the broader perspective of the 1970 report.

Because users of this report will almost certainly be referring to JPL TR 32-1329 (Ref. 8), it seems appropriate to include here a few technical remarks that may contribute to the usefulness of the latter, and to note as well those persistent minor errors in the earlier text which have been brought to the author's attention. All page references following refer to JPL TR 32-1329. 
(1) Page 17 indicates that the deformations $\mathbf{u}^{s}$ and $\beta^{s}$ are to be measured relative to some "nominal undeformed state." The word "undeformed" is unnecessarily restrictive, and can be stricken without impairing the validity of all that follows.

(2) Page 22 indicates that the inertia matrix $I^{S}$ is diagonal. This restriction is anticipated on page 17 with the remark "it is convenient to select basis $\{a\}$ and the individual mass-center principal axis bases of $A_{1}, \cdots, A_{n}$ to be identical." This restriction is actually used only twice in the entire report, and the restriction is readily removed. The proof of Eq. (88) on page 26 is easily generalized for nondiagonal $I^{S}$, and the result stands. On page 28 the fifth term in Eq. (91) is expanded in scalar form, assuming diagonal $I^{S}$, and the presence of off-diagonal terms in $I^{S}$ would complicate this expansion. However, the added terms have no consequences beyond page 28 .

(3) Eq. (128), page 36, simplifies with the recognition of certain identities. The term $\approx \Sigma m^{S}\left[2\left(R+r^{S}\right)^{T} u^{S} E\right] \omega$ is identically zero, since $\approx \omega=0$. More significantly, the coefficients of $u^{s}$ can be combined as follows:

$$
\begin{aligned}
& \Sigma m^{S}\left[2\left(R+r^{S}\right)^{T} \dot{u}^{S E}-\left(R+r^{S}\right) \dot{u}^{S^{T}}-\dot{u}^{S}\left(R+r^{S}\right)^{T}\right] \omega \\
& +(\tilde{\omega} R)^{\sim} \Sigma m^{s} \dot{u}^{s}+\widetilde{R} \widetilde{\omega} \Sigma m^{s} \dot{u}^{S}+\left(\widetilde{\omega} \Sigma r^{S}\right)^{\sim} m^{s} \dot{u}^{s}
\end{aligned}
$$

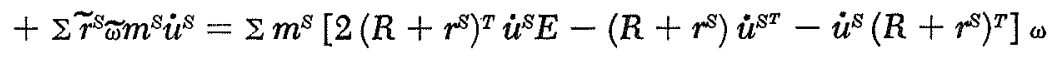

$$
\begin{aligned}
& +[\widetilde{\omega} \widetilde{R}-\widetilde{R} \widetilde{\omega}+\widetilde{R} \widetilde{\omega}] \Sigma m^{s} \dot{u}^{s}+\left[\widetilde{\omega} \Sigma \widetilde{r}^{S}-\Sigma \widetilde{r}^{S} \widetilde{\omega}+\Sigma \widetilde{r}^{\mathrm{s}} \widetilde{\omega}\right] m^{s} \dot{u}^{\mathrm{s}} \\
& =\Sigma_{1} m^{S}\left[2\left(R+r^{S}\right)^{T} \dot{u}^{S} \omega-\left(R+r^{S}\right) \dot{u}^{S^{T}}{ }_{\omega}-\dot{u}^{S}\left(R+r^{S}\right)^{T} \omega\right] \\
& +R \Sigma m^{S} \dot{u}^{S^{T}} \omega-\Sigma m^{S} \dot{u}^{S} R^{T} \omega+\Sigma m^{S} r^{S} u^{S^{T}}{ }_{\omega}-\Sigma m^{S} \dot{u}^{S} r^{S^{T}} \omega \\
& =\Sigma m^{S}\left[2\left(R+r^{S}\right)^{T} \dot{u}_{\omega}^{S}-2 \dot{u}^{S}\left(R+r^{S}\right)^{T} \omega\right] \\
& =2 \Sigma m^{s}\left(R+r^{s}\right) \sim \widetilde{\omega} \dot{u}^{s}
\end{aligned}
$$

(4) The simplifications indicated in (3) for Eq. (128) provide corresponding simplifications of Eq. (129).

(5) Note the text corrections listed on the following pages. 


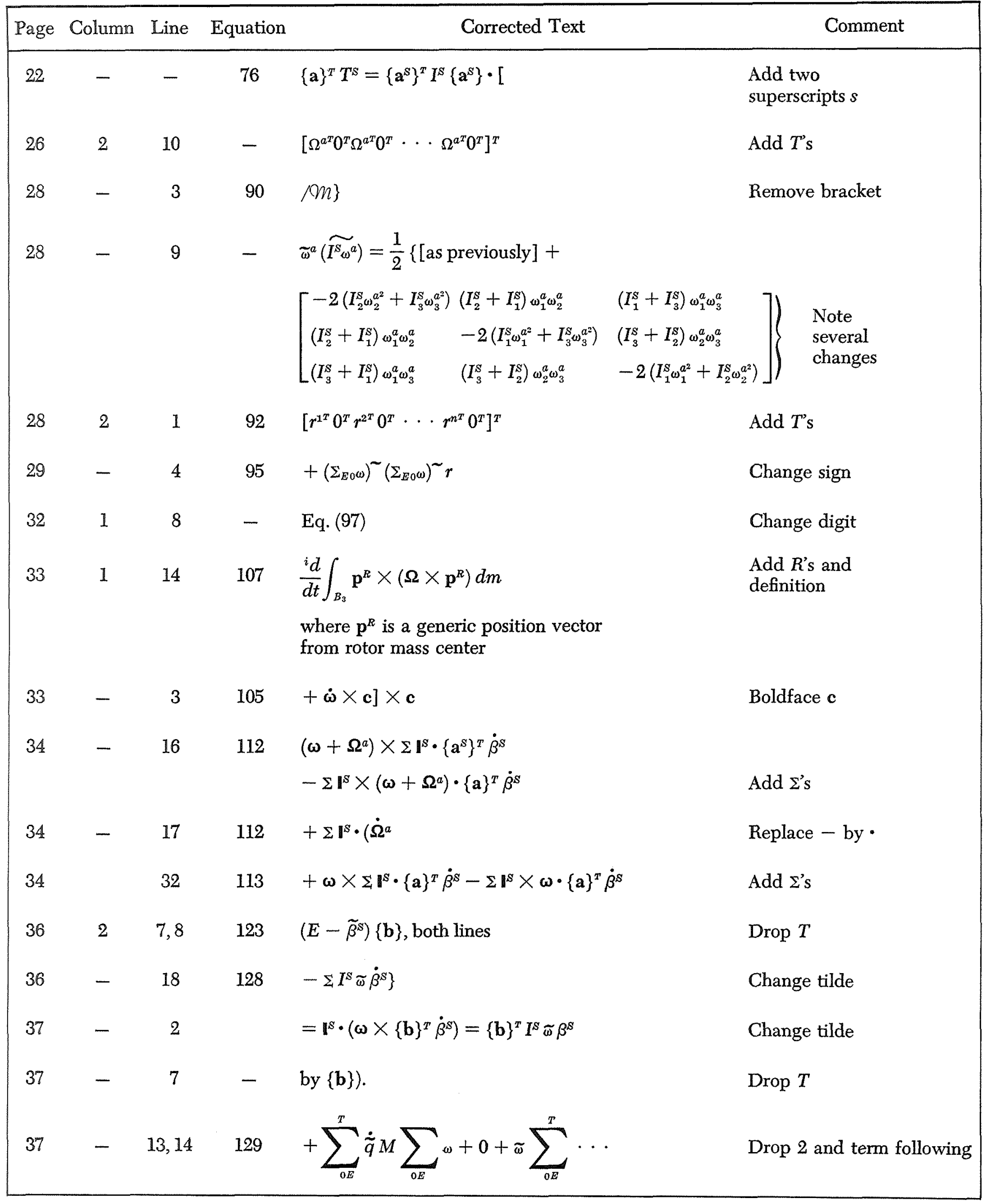




\begin{tabular}{|c|c|c|c|c|c|}
\hline Page & Column & Line & Equation & Corrected Text & Comment \\
\hline 38 & 2 & 20 & 137 & $\widetilde{\omega}=-\dot{\Theta} \Theta^{T}=\Theta \dot{\Theta}^{T}$ & Change signs \\
\hline 40 & - & 4 & 139 & $+\left(\Sigma_{E 0} \omega\right) \sim\left(\Sigma_{E 0} \omega\right) \sim r$ & Change sign and tilde \\
\hline 47 & 2 & 11 & 174 & {$\left[\begin{array}{c}\psi^{m} \\
----\end{array}\right]$} & \\
\hline & & & & $\begin{array}{l}{\left[-\gamma^{m} \sigma_{m}\right.} \\
{\left[\gamma^{m}\right]}\end{array}$ & $\begin{array}{l}\text { Interchange } \\
\text { symbols and } \\
\text { change sign }\end{array}$ \\
\hline 49 & - & - & 192 & {$\left[\begin{array}{l|l} & -\gamma \sigma \\
\hdashline \sigma\end{array}\right]$} & $\begin{array}{l}\text { Note implications on } \\
\text { pp. } 49-51\end{array}$ \\
\hline 50 & 2 & 2 & 196 & $-\sigma_{m} C_{m} \sin \sigma_{m} t+\sigma_{m} D_{m} \cos \sigma_{m} t$ & Drop inverses \\
\hline 61 & 1 & 3 & 267 & $m^{A} \tilde{p} \quad$ (twice) & Drop superscript $A$ \\
\hline 61 & 1 & 6 & - & basis $\{\mathbf{b}\}$ & $\{\mathbf{a}\} \rightarrow\{\mathbf{b}\}$ \\
\hline 68 & 2 & 42 & 286 & $\bar{\sigma}^{2} \bar{\eta}$ & Add bar to $\sigma^{2}$ \\
\hline 70 & 2 & 39 & 300 & {$[E-R(s) F(s)] \theta(s)$} & Change $I$ to $E$ \\
\hline 80 & - & 31 & & Wittenburg & Change spelling \\
\hline
\end{tabular}




\begin{abstract}
The increasingly common practice of idealizing a spacecraft as a collection of interconnected rigid bodies to some of which are attached linearly elastic flexible appendages leads to equations of motion expressed in terms of a combination of discrete coordinates describing the arbitrary rotational motions of the rigid bodies and distributed or modal coordinates describing the small, time-varying deformations of the appendages; such a formulation is said to employ a hybrid system of coordinates. In the present paper the existing literature is extended to provide hybrid coordinate equations of motion for a finite element model of a flexible appendage attached to a rigid base undergoing unrestricted motions, and some of the advantages of the finite element approach are noted. Transformations to the modal coordinates appropriate for the general case and various special cases are provided.
\end{abstract}




\section{Finite Element Appendage Equations for Hybrid Coordinate Dynamic Analysis}

\section{Introduction}

A typical modern spacecraft consists of structural subsystems, some essentially rigid and others extremely flexible, interconnected often in a time-varying manner, with relative motions frequently prescribed by nonlinear automatic control systems. Such vehicles may in whole or in part be spinning, they may be expected to undergo arbitrary large changes in inertial orientation, and they may be subjected to external forces due to environmental interaction and due to the actuation of attitude control devices. It has become necessary, largely for the purpose of attitude control system design and analysis, to devise methods of dynamic analysis which combine the generalities of nonlinearity and unrestricted motions provided by the representation of the vehicle as a collection of interconnected discrete rigid bodies (Refs. 1 and 2) with the computational efficiency afforded by the use of modal coordinates to describe the vehicle normal mode deformations (Refs. 3-5). The result is a procedure which employs discrete coordinates to describe the unrestricted motions of those structural subsystems idealized as rigid bodies, in combination with distributed or modal coordinates to describe the time-varying deformations of those structural subsystems idealized as flexible elastic appendages. This method is called the hybrid coordinate approach to space vehicle dynamic simulation.

Within the framework of the hybrid coordinate methods, three alternative approaches to the initial mathematical modeling of flexible appendages can be distinguished: (1) appendages are idealized as collections of small rigid bodies interconnected by massless elastic structure (Refs. 6-8); (2) appendages are treated as elastic continua (Refs. 9-11); and (3) appendages are modeled as collections of finite elastic elements possessing mass, interconnected at nodes where mass may or may not be concentrated. In every case, the formulation of equations of motion for the appendage deformations is followed by a transformation to distributed or modal coordinates for the appendages, so that in the final system of equations of motion the initial mathematical model adopted for the appendages is obscured; indeed, one can formulate the system equations in terms of appendage modal coordinates without confronting the question of the origin of these coordinates in the equations of motion of a particular mathematical model of the appendages (Ref. 12). 
The first of the three approaches to appendage modeling has been developed to the point of providing information useful for the design of attitude control systems of very complex modern spacecraft (Refs. 13-15), and the second approach has proven to have practical value when the appendages are amenable to idealization as elastic beams (Refs. 9-11). It is the purpose of this paper to provide the equations required by the third approach, and to identify features of these equations which make the resulting finite element formulation superior in some applications to the two alternatives previously developed, and then to develop and evaluate procedures for obtaining transformations to modal coordinates.

\section{Appendage Idealization}

Any portion of a vehicle which can reasonably be idealized as linearly elastic and for which "small" oscillatory deformations may be anticipated (perhaps in combination with large steady-state deformations) is called a flexible appendage.

A Alexible appendage is idealized as a finite collection of $\delta$ numbered structural elements, with element number $s$ having $n_{s}$ points of contact in common with neighboring elements or a supporting rigid body, $s=1, \cdots, \delta$. Each contact point is called a node, and at each of the $n$ nodes there may be located the mass center of a rigid body (called a nodal body), but the elastic structural elements may also have distributed mass. In the final equations, the element masses can be suppressed to obtain the results of Ref. 8, or the nodal masses can be suppressed if the physical system permits such an idealization.

Figure 1 is a schematic representation of an appendage (enclosed by dashed lines) attached to a rigid body $b$ of a spacecraft, which may consist of several interconnected rigid bodies and Alexible appendages. A typical four-node element of the appendage is shown in three configurations of interest: (1) prior to structural deformation, (2) subsequent to steady-state deformation, induced perhaps by spin, and (3) in an excited state, experiencing both oscillatory deformations and steady-state deformations.

The point $\mathscr{Q}$ of body $b$ is selected as an appendage attachment point. The dextral, orthogonal unit vectors $b_{1}, b_{2}, b_{3}$ are fixed relative to $b$, and the dextral, orthogonal unit vectors $\mathbf{a}_{1}, \mathbf{a}_{2}, \mathbf{a}_{3}$ are so defined that the flexible appendage undergoes structural deformations relative to a reference frame ${ }_{a}$ established by point $\&$ and vectors $\mathfrak{a}_{1}, \mathbf{a}_{2}, \mathfrak{a}_{3}$. Gross changes in the relative orientation of $a$

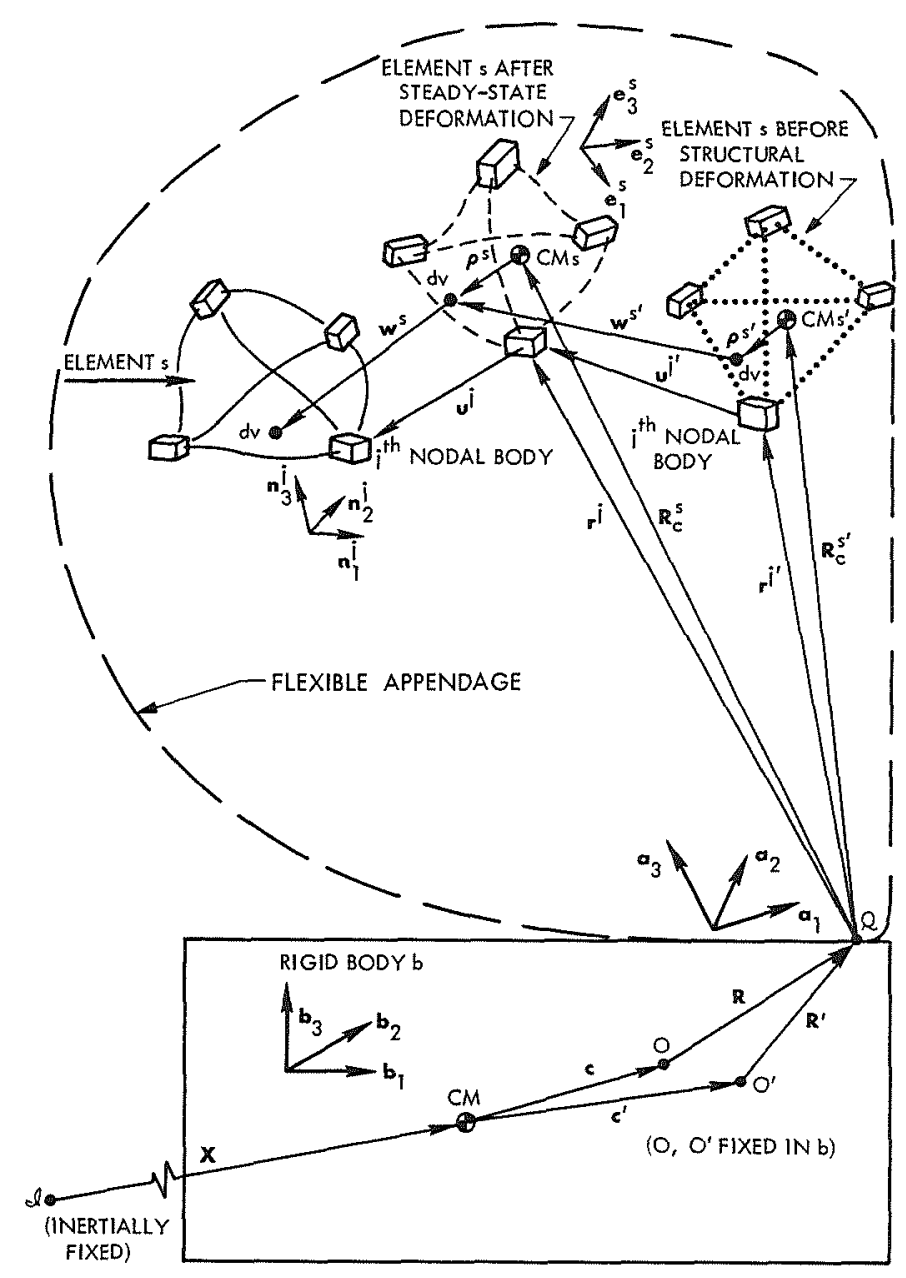

Fig. 1. Appendage idealization

and $b$ are permitted, in order to accommodate scanning antennas and such devices; this is accomplished by introducing the time-varying direction cosine matrix $C$ relating $a_{\alpha}$ to $b_{\alpha}(\alpha=1,2,3)$ by

$$
\left\{\begin{array}{l}
\mathbf{a}_{1} \\
\mathbf{a}_{2} \\
\mathbf{a}_{3}
\end{array}\right\}=\left[\begin{array}{lll}
c_{11} & c_{12} & c_{13} \\
c_{21} & c_{22} & c_{23} \\
c_{31} & c_{32} & c_{33}
\end{array}\right]\left\{\begin{array}{l}
\mathbf{b}_{1} \\
\mathbf{b}_{2} \\
\mathbf{b}_{3}
\end{array}\right\}
$$

or, in more compact notation, by

$$
\{\mathbf{a}\}=C\{\mathbf{b}\}
$$

The equations of motion to follow permit arbitrary motion of $b$ and arbitrary time variation in $C$, although practical application of the results requires that the iner- 
tial angular velocity of $b$ and the angular velocity of $a$ relative to $b$ remain in the neighborhood of constant values. These angular velocities will not emerge as solutions of equations to be derived here; the complete dynamic simulation must involve equations of motion of the total vehicle and each of its subsystems, as well as differential equations characterizing necessary control laws for automatic control systems, and only the differential equations of appendage deformation are to be developed here.

As shown in Fig. 1, appendage deformations are described in terms of two increments, one steady-state and the other oscillatory. This separation is necessary because in formulating the equations of motion for the small oscillatory deformations of primary interest here one must characterize the elastic properties of the appendage with a stiffness matrix, and the elements of this matrix are influenced by the structural preload associated with steadystate deformations, as induced, for example, by spin.

The $j$ th nodal body experiences due to steady-state structural deformation the translation $\mathbf{u}^{j \prime}=u_{\alpha}^{j \prime} \mathbf{a}_{\alpha}$ (summation convention) of its mass center and a rotation characterized by $\beta_{1}^{j \prime}, \beta_{2}^{j \prime}, \beta_{3}^{j \prime}$ for sequential rotations about axes parallel to $\mathbf{a}_{1}, \mathbf{a}_{2}, \mathbf{a}_{3}$. The steady-state deformations of a typical element are represented by the function $\mathbf{w}^{\prime}$, which is related to the corresponding nodal deformation by the procedures of finite element analysis. The task of solving for the steady-state deformations of appendages on a vehicle with constant angular velocity is mathematically identical to a static deflection problem. Because, at least formally, large deflections and resulting nonlinearities are to be accommodated, this task is not trivial, but in this paper it is assumed accomplished, so that steady-state deformations and structural loads associated with nominal vehicle rotation are assumed known.

Attention is to focus here on the small, time-varying deformations of appendages induced by transient loads or deviations from nominal vehicle motion. The $j$ th nodal body experiences the translation $\mathbf{u}^{j}=\boldsymbol{u}_{\alpha}^{j} \mathbf{a}_{\alpha}$ and the rotation $\boldsymbol{\beta}^{j}=\beta_{\alpha}^{j} \mathbf{a}_{\alpha}$ (small angle approximation) in addition to the previously described steady-state deformations. The oscillatory part of the deformation of a generic element is represented by the vector function w. (Should it become necessary to deal with such deformations for more than one element simultaneously, the notation $\mathrm{w}^{8}$ is employed for element s.) The quantities $\mathbf{u}^{j}, \boldsymbol{\beta}^{j}(j=1, \cdots, n)$ and $\mathrm{w}^{s}(s=1, \cdots, \delta)$ or their scalar components are referred to as variational deformations.
For convenience in calculations it is often desirable to introduce for each finite element in its steady-state condition a local coordinate system; this may be accomplished by defining a set of dextral, orthogonal unit vectors $\mathbf{e}_{1}, \mathbf{e}_{2}, \mathbf{e}_{3}$, an origin $\mathcal{Q}$, and a corresponding set of axes $\dot{\xi}, \eta, \zeta$. (Superscripts are appended to each of these symbols should it become necessary to distinguish the particular element.) The local vector basis is then related to the appendage global vector basis $a_{1}, a_{2}, a_{3}$ by a constant direction cosine matrix $\bar{C}$, as in

$$
\left\{\begin{array}{l}
\mathbf{e}_{1} \\
\mathbf{e}_{2} \\
\mathbf{e}_{3}
\end{array}\right\}=\bar{C}\left\{\begin{array}{l}
\mathbf{a}_{1} \\
\mathbf{a}_{2} \\
\mathbf{a}_{3}
\end{array}\right\} \quad \text { or }\{\mathbf{e}\}=\bar{C}\{\mathbf{a}\}
$$

The vector function $\mathbf{w}$ is most conveniently expressed in terms of local coordinates and the local vector basis; the $(3 \times 1)$ matrix function $w$ defined by

$$
\mathbf{w}=\bar{w}_{\alpha} \mathbf{e}_{\alpha}=\{\mathbf{e}\}^{T} \bar{w}
$$

represents $w$ in the local basis, whereas the $(3 \times 1)$ matrix function $w$ defined by

$$
\mathbf{w}=w_{\alpha} \mathbf{a}_{\alpha}=\{\mathbf{a}\}^{T} w
$$

represents $\mathbf{w}$ in the global basis. Similar notation distinguishes the vector bases of all matrices representing Gibbsian vectors.

An important aspect of the appendage idealization is the assumption, to be incorporated in the following section, that the deformations of each finite element can be represented as a function only of the deformations of its nodes, and that the nature of that function can be imposed a priori.

\section{Finite Element Equations of Motion}

Having adopted an appendage idealization, one can proceed formally to derive its equations of motion. Since it is the variational nodal deformations $\mathbf{u}^{j}$ and $\beta^{j}(j=1, \cdots, n)$ which represent the appendage unknowns, the equations of motion of the appendage ultimately consist of the $6 n$ scalar second-order differential equations of motion for the $n$ nodal bodies. The present section, however, has the 
intermediate objective of providing an expression relating the variational deformation function $w$ of a finite element to the variational deformations at its nodes, and in terms of this relationship providing expressions for the forces and torques applied to the nodal bodies by the adjacent finite elements.

Rather than attempt to work with the infinite number of degrees of freedom of the element as a continuous system, one can avoid introducing any additional degrees of freedom attributable to element mass by assigning to $\mathrm{w}(\xi, \eta, \xi)$ a functional structure permitting its representation in terms of the $6 \mathrm{n}$ scalars defining the translational and rotational displacements due to oscillatory deformations at its $n$ nodes. ${ }^{1}$ Although much is left to the discretion of the analyst in choosing an expression for the function $w(\xi, \eta, \zeta)$, it is required for present purposes that this expression involve $6 n$ scalars $\Gamma_{1}, \cdots, \Gamma_{87}$, matching in number the unknown deformational displacements at the $n$ nodes of the element. Typically, polynomials in the Cartesian coordinates $\xi, \eta, \zeta$ are chosen, with $\Gamma_{1}, \cdots, \Gamma_{6 n}$ providing the coefficients. In matrix form, the indicated relationship is written

$$
\bar{w}=P \Gamma
$$

where $\bar{w}$ is defined by Eq. (3), $\Gamma \triangleq\left[\Gamma_{1} \Gamma_{2} \cdots \Gamma_{6 \eta l}\right]^{T}$, and $P$ is a $(3 \times 6 n)$ matrix establishing the assumed structure of the deformational displacement function. For Eq. (5) to provide an appropriate polynomial relationship, $P$ is written in the form

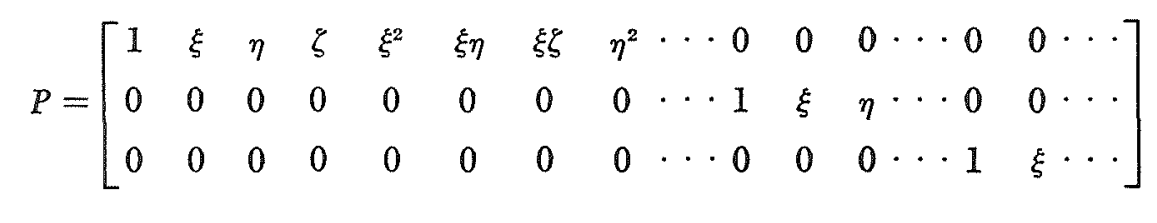

If the tetrahedral element of Fig. 1 is adopted and the rotational degrees of freedom at the nodes are abandoned, permitting only particles to be placed at the nodes, then the element has $(4 \times 3)=12$ nodal deformational displacements, the dimension of $P$ is $(3 \times 12)$, and $P$ may be chosen to consist of linear functions in the pattern of Eq. (6). If, however, rotational nodal deformations are retained (as in Fig. 1), the dimension of $P$ is $(3 \times 24)$, and some choice of nonlinear functions is required (perhaps in each row of $P$ there would appear eight of the 10 terms of a quadratic function, with two judiciously suppressed).

Equation (5) applies throughout a given finite element, and hence it applies at the element nodes; if the $j$ th node of the appendage is a node of the element in question, with local coordinates $\xi_{j}, \eta_{j}, \zeta_{j}$, the nodal displacement $\mathbf{u}^{j}$ as represented by the matrix $\bar{u}^{j}$ in the local basis is from Eq. (5) given by

$$
\bar{u}^{j}=\bar{w}\left(\xi_{j}, \eta_{j}, \zeta_{j}\right)=P\left(\xi_{j}, \eta_{j}, \zeta_{j}\right) \Gamma
$$

\footnotetext{
${ }^{1}$ The symbol $n_{s}$ represents the number of nodes of element $s$, but
} the symbol $n$ will be used for a generic element. and the rotation $\beta^{j}$ is represented in the local basis by the matrix

$$
\bar{\beta}^{j}=\left.\frac{1}{2} \widetilde{\nabla} \bar{w}\right|_{\xi_{j, \eta_{j}, \xi_{j} j}}=\left.\frac{1}{2} \widetilde{\nabla} P\right|_{\xi_{j,}, \eta_{j}, \zeta_{j} j} \Gamma
$$

where

$$
\widetilde{\nabla} \triangleq\left[\begin{array}{ccc}
0 & -\partial / \partial \xi & \partial / \partial \eta \\
\partial / \partial \zeta & 0 & -\partial / \partial \xi \\
-\partial / \partial \eta & \partial / \partial \xi & 0
\end{array}\right]
$$

Equations (7) and (8), written for each of the $n$ nodes of a given finite element, furnish $6 \bigcap$ scalar equations, sufficient to permit solution for $\Gamma_{1}, \cdots, \Gamma_{\theta n}$ in terms of the $6 n$ nodal deformations. If the nodal numbers of the element are designated $k, i, \cdots, j$ (no sequence implied), and a $(6 n \times 1)$ matrix $\bar{y}$ is introduced to represent, in the local basis of the element, all of the deformational displacements of adjacent nodes, one can construct the matrix equation

$$
\bar{y}=F \Gamma
$$


with

$$
y \triangleq\left[\begin{array}{c}
\bar{u}^{k} \\
\bar{\beta}^{k} \\
\bar{u}^{i} \\
\bar{\beta}^{i} \\
\vdots \\
\bar{u}^{j} \\
\bar{\beta}^{j}
\end{array}\right] \quad F \triangleq\left[\begin{array}{c}
\left.P\right|_{k} \\
\left.\frac{1}{2} \widetilde{\nabla} P\right|_{k} \\
\left.P\right|_{i} \\
\left.\frac{1}{2} \widetilde{\nabla} P\right|_{i} \\
\vdots \\
\left.P\right|_{j} \\
\left.\frac{1}{2} \widetilde{\nabla} P\right|_{j}
\end{array}\right]
$$

where the notation $\left.\right|_{j}$ implies evaluation at $\xi_{j}, \eta_{j}, \zeta_{j}$, etc.

Substituting the inverse of Eq. (9) into Eq. (5) yields

$$
\bar{w}=P F^{-1} \bar{y}
$$

thus establishing the relationship between nodal deformations and the deformations distributed throughout the element. The $(3 \times 60)$ matrix $P F^{-1}$, which appears frequently in what follows, is designated $W$, permitting $\bar{w}$ to be written

$$
\bar{w}=W \bar{y}
$$

With full knowledge of the variational deformation field $\bar{w}$ throughout the element, one can obtain an expression for the variational strain field, represented in the local vector basis by $\epsilon_{\alpha \gamma}, \alpha, \gamma=1,2,3$. This step requires straindisplacement relationships. When large displacements are considered, as they must be if a steady-state strain due to appendage preload is to be calculated, the nonlinear version of the strain-displacement equations is appropriate. This results in substantial analytical complexity, normally circumvented by a process of incremental use of straindisplacement equations linearized about different displacement states. Nonlinearities in the strain-displacement equations are avoided in the present analytical formulation for the solution for small, variational, time-varying deformational displacements by linearizing the straindisplacement equations about the state established by the steady-state preload. Thus the incremental or variational strains in the element beyond any steady-state strains (which will be called $\bar{\epsilon}_{a \gamma}^{\prime} ; \alpha, \gamma=1,2,3$ ) can always be re- lated to small variations $\bar{w}_{1}, \bar{w}_{2}, \bar{w}_{3}$ in displacements with an equation of the form

$$
\left[\begin{array}{l}
\bar{\epsilon}_{11} \\
\bar{\epsilon}_{22} \\
\bar{\epsilon}_{33} \\
\bar{\epsilon}_{12} \\
\bar{\epsilon}_{23} \\
\bar{\epsilon}_{13}
\end{array}\right]=D\left[\begin{array}{c}
\bar{w}_{1} \\
\bar{w}_{2} \\
\bar{w}_{3}
\end{array}\right]
$$

or $\bar{\epsilon}=D \bar{w}$, which becomes

$$
\bar{\epsilon}=D W \bar{y}
$$

and when these are small deformationall displacements $\bar{w}_{1}, \bar{w}_{2}, \bar{w}_{3}$ corresponding to orthogonal axes $\xi, \eta, \zeta$, Eq. (12) takes the form

$$
\left[\begin{array}{c}
\bar{\epsilon}_{11} \\
\bar{\epsilon}_{22} \\
\bar{\epsilon}_{33} \\
\bar{\epsilon}_{12} \\
\bar{\epsilon}_{23} \\
\bar{\epsilon}_{31}
\end{array}\right]=\left[\begin{array}{ccc}
\partial / \partial \xi & 0 & 0 \\
0 & \partial / \partial \eta & 0 \\
0 & 0 & \partial / \partial \zeta \\
\partial / \partial \eta & \partial / \partial \xi & 0 \\
0 & \partial / \partial \zeta & \partial / \partial \eta \\
\partial / \partial \zeta & 0 & \partial / \partial \xi
\end{array}\right]\left[\begin{array}{l}
\bar{w}_{1} \\
\bar{w}_{2} \\
\bar{w}_{3}
\end{array}\right]
$$

In addition to the variational strain matrix $\bar{\epsilon}$ above, one may define a steady-state strain matrix $\bar{\epsilon}^{\prime}$ with six elements chosen from $\bar{\epsilon}_{\alpha \gamma}^{\prime}(\alpha, \gamma=1,2,3)$, and also a strain matrix $\bar{\epsilon}_{\tau}$ that would result as a consequence of any deviations from the steady-state thermal condition of the structural appendage. If the deviation from the steady-state temperature at a given point of the element is designated $\tau$, the variational thermal strain $\bar{\epsilon}_{\tau}$ becomes

$$
\bar{\epsilon}_{\tau}=\bar{\alpha} \tau\left[\begin{array}{llllll}
1 & 1 & 1 & 0 & 0 & 0
\end{array}\right]^{T}
$$

where the scalar $\bar{\alpha}$ is the coefficient of thermal expansion of the element material. When finite element heat transfer equations are introduced to augment the dynamical equations sought here, the distribution of temperature $\tau(\xi, \eta, \zeta)$ in each element would be assumed to have a simple functional dependence on the nodal temperatures, which become additional unknowns. 
The increment $\bar{\sigma}$ in the stress matrix beyond the steady-state value $\bar{\sigma}^{\prime}$ is related for an elastic material to the difference in the total variational strain and the variational thermal strain by

$$
\left[\begin{array}{c}
\bar{\sigma}_{11} \\
\bar{\sigma}_{22} \\
\bar{\sigma}_{33} \\
\vec{\sigma}_{12} \\
\bar{\sigma}_{23} \\
\bar{\sigma}_{31}
\end{array}\right]=\frac{E}{(1+v)(1-2 v)}\left[\begin{array}{cccccc}
(1-v) & v & v & 0 & 0 & 0 \\
v & (1-v) & v & 0 & 0 & 0 \\
v & v & (1-v) & 0 & 0 & 0 \\
0 & 0 & 0 & (1-2 v) / 2 & 0 & 0 \\
0 & 0 & 0 & 0 & (1-2 v) / 2 & 0 \\
0 & 0 & 0 & 0 & 0 & (1-2 v) / 2
\end{array}\right]\left[\begin{array}{c}
\bar{\epsilon}_{11}-\bar{\alpha} \tau \\
\bar{\epsilon}_{22}-\bar{\alpha} \tau \\
\bar{\epsilon}_{33}-\bar{\alpha} \tau \\
\bar{\epsilon}_{12} \\
\bar{\epsilon}_{23} \\
\bar{\epsilon}_{31}
\end{array}\right]
$$

where $E$ is Young's modulus and $\nu$ is Poisson's ratio. Symbolically, Eq. (16) may be written

$$
\bar{\sigma}=S \bar{\epsilon}-\bar{\sigma}_{\tau}
$$

which with Eq. (13) becomes

$$
\bar{\sigma}=S D W \bar{y}-\bar{\sigma}_{\tau}
$$

The $(6 \times 60 n)$ matrix $S D W$ is sometimes called the element stress matrix.

Variational stresses and strains are related to nodal variational displacements in Eqs. (18) and (13) respectively. This information can be used in conjunction with the work-energy equation and the virtual displacement concept to obtain expressions for forces and torques that must be applied to the element at the nodes in order to balance the applied loads while sustaining the inertial accelerations associated with nodal accelerations by Eq. (11). Since equal and opposite forces and torques are applied by the elements to the nodal bodies for which equations of motion are to be written in the next section, these expressions are the primary immediate objective.

For static equilibrium of a mechanical system, the work (1)* accomplished by external forces in the course of a virtual displacement $\bar{y}^{*}$ equals the energy $\mathcal{U}^{*}$ stored as strain energy in the deforming element; this equality is preserved for nondissipative dynamical systems in motion if to the external forces one adds the inertial "force," which for a differential element of volume $d v$ at point $p$ is $-\mathbf{A} \mu d v$, where $\mathbb{A}$ is the inertial acceleration of the point $p$, and $\mu$ is the mass density at $p$. In general, then, the external "forces" doing work include the inertial "forces," the forces and torques applied to the element at its nodes, the body forces (designated by the matrix function $\bar{G}(\xi, \eta, \zeta)$ in the local basis), and the surface forces. In spacecraft applications it is usually sufficient to eliminate the surface loads from participation in 2$)^{2 *}$ by distributing them to the nodes (as indeed may often be appropriate for the body forces).

For the finite element designated $s$, let the $\left(6 n_{s} \times 1\right)$ matrix $\overline{L^{s}}$ be introduced as

$$
\bar{L}^{s} \triangleq\left[\begin{array}{c}
\bar{F}^{k s} \\
\bar{T}^{k s} \\
\vdots \\
\bar{F}^{s s} \\
\bar{T}^{j s}
\end{array}\right]
$$

where $\bar{F}^{k s}$ and $\bar{T}^{k s}$ are $(3 \times 1)$ matrices in the local (element) vector basis, respectively representing force and torque applied by the $k$ th nodal body to the $s$ th element, and similarly for all $n_{s}$ nodes of the $s$ th finite element. Thus the work (2)* associated with a virtual displacement of the nodes of a generic element relative to $a$ becomes

$$
Q \mathcal{S}^{*}=\bar{y}^{* T} \bar{L}+\int \bar{w}^{* T} \overline{\mathrm{G}} d v-\int \bar{w}^{* T} \bar{A} \mu d v
$$

where $\bar{A}$ is the $(3 \times 1)$ matrix representing $\mathbf{A}$ in the local vector basis. With Eq. (11), the work expression becomes

$$
Q \mathcal{S}^{* *}=\bar{y}^{* T}\left[\bar{L}+\int W^{x}\left(\bar{G}-\bar{A}_{\mu}\right) d v\right]
$$


The strain energy $\mathcal{U}^{*}$ is by virtue of Eqs. (18), (13), and (1I) given by

$$
\begin{aligned}
U^{*} & =\int \bar{\epsilon}^{* T}\left(\bar{\sigma}+\bar{\sigma}^{\prime}\right) d v=\int \bar{w}^{* T} D^{T}\left(\operatorname{SDW} \bar{y}^{*}-\bar{\sigma}_{\tau}+\bar{\sigma}^{\prime}\right) d v \\
& =\bar{y}^{* T} \int W^{T} D^{T} S D W d v \bar{y}-\bar{y}^{* T} \int W^{T} D^{T} \bar{\sigma}_{\tau} d v+\bar{y}^{* T} \int W^{T} D^{T} \bar{\sigma}^{\prime} d v
\end{aligned}
$$

Equating $\mathcal{U}^{*}$ and ()$^{)^{*}}$, dismissing the arbitrary premultiplier $\bar{y}^{* T}$, and solving for $\bar{L}$ furnishes

$$
\bar{L}=\int W^{T} D^{T} S D W d v \bar{y}+\int W^{r}\left(\bar{A}_{\mu}-\bar{G}-D^{r} \bar{\sigma}_{\tau}\right) d v+\int W^{T} D^{r} \bar{\sigma}^{\prime} d v
$$

Note that the last term in Eq. (22) contributes only to the steady-state value of $\bar{L}$.

Equation (22) is in useful form only when the inertial acceleration matrix $\bar{A}$ is written in terms of the nodal deformation matrix $\bar{y}$ and those functions which define the arbitrary motion of the base 6 to which the appendage is attached. This is most readily accomplished first in terms of the corresponding Gibbsian vector $\mathbf{A}$, which by definition is available in terms of the symbols of Fig. 1 as

$$
\mathbb{A} \triangleq \frac{{ }^{i} d^{2}}{d t}\left(\mathbf{X}+\mathbf{c}+\mathbf{R}+\mathbf{R}_{c}+\mathbf{p}+\mathbf{w}\right)
$$

where the presuperscript $i$ denotes an inertial reference frame for vector differentiation, and the chain of vectors in parentheses is a single vector locating a differential element of volume in a finite element with respect to an inertially fixed point $\delta$. If it should be necessary to identify the particular finite element to which Eq. (23) is being applied, the corresponding numerical superscript can be attached to the vectors $\mathbf{A}, \mathbf{R}_{c}, \boldsymbol{\rho}$, and $\mathbf{w}$.

Since a matrix formulation is ultimately required, $(3 \times 1)$ matrices are defined for each of the vectors in Eq. (23) in terms of the most convenient vector basis. In terms of the vector arrays $\{\mathbf{b}\},\{\mathbf{a}\}$, and $\{\mathbf{e}\}$ of Eqs. (1) and (2), and the new array $\{$ i $\}$ of inertially fixed unit vectors related to $\{b\}$ by

$$
\{\mathbf{b}\}=\Theta\{\mathbf{i}\}
$$

the vectors in Eq. (23) may be written

$$
\begin{aligned}
& \mathbf{X} \triangleq\{\mathbf{i}\}^{T} X \\
& \mathbf{c} \triangleq\{\mathbf{b}\}^{T} c \\
& \mathbf{R} \triangleq\{\mathbf{a}\}^{T} R \\
& \mathbf{R}_{c} \triangleq\{\mathbf{a}\}^{T} R_{c} \\
& \mathbf{\rho} \triangleq\{\mathbf{e}\}^{T} \bar{\rho}=\{\mathbf{a}\}^{T} \rho \\
& \mathbf{w} \triangleq\{\mathbf{e}\}^{T} \bar{w}=\{\mathbf{a}\}^{T} w
\end{aligned}
$$

thereby defining $X, c, R, R_{c}, \bar{\rho}, \rho, \bar{w}$, and $w$.

The inertial reference frame differentiations in Eq. (23) are facilitated by the identity

$$
\frac{{ }^{g} d}{d t} \mathbf{V}=\frac{{ }^{f} d}{d t} \mathbf{V}+\boldsymbol{\omega}^{\prime \prime} \times \mathbf{V}
$$

applicable to any vector $\mathrm{V}$ and any two references frames $f$ and $g$, where $\omega^{t g}$ is the angular velocity of $f$ relative to $g$. 
With repeated use of Eq. (26), Eq. (23) takes the form

$$
\begin{aligned}
A= & \{\mathbf{i}\}^{T} \ddot{X}+\{\mathbf{b}\}^{T} \ddot{c}+2 \omega \times\{\mathbf{b}\}^{T} \dot{c}+\dot{\omega} \times\{\mathbf{b}\}^{T^{T}} c \\
& +\omega \times\left(\omega \times\{\mathbf{b}\}^{T} c\right) \\
& +\dot{\omega} \times\{\mathbf{b}\}^{T} R+\omega \times\left(\boldsymbol{\omega} \times\{\mathbf{b}\}^{T} R\right) \\
& +\dot{\omega^{a}} \times\{\mathbf{a}\}^{T} R_{c}+\omega^{a} \times\left(\omega^{a} \times\{\mathbf{a}\}^{T} R_{c}\right) \\
& +\dot{\omega}^{a} \times\{\mathbf{a}\}^{T} \rho+\omega^{a} \times\left(\omega^{a} \times\{\mathbf{a}\}^{T} \rho\right) \\
& +\{a\}^{T} \ddot{w}+2 \omega^{a} \times\{\mathbf{a}\}^{T} \dot{w}+\dot{\omega}^{a} \times\{a\}^{T} w \\
& +\omega^{a} \times\left(\omega^{a} \times\{\mathbf{a}\}^{T} w\right)
\end{aligned}
$$

where $\omega$ and $\omega^{a}$ are the inertial angular velocities of $b$ and a respectively (so that in the more explicit notation of Eq. 26 one would have $\omega \triangleq \omega^{b i}$ and $\left.\omega^{a} \triangleq \omega^{a i}\right)$. Equation (2) can be used to replace $w$ and $\rho$ in Eq. (27) by $\bar{w}$ and $\vec{\rho}$ respectively, and with the introduction of matrices $\omega$ and $\omega^{a}$ defined by

$$
\boldsymbol{\omega}=\{\mathbf{b}\}^{T} \omega \quad \boldsymbol{\omega}^{a}=\{\mathbf{a}\}^{T} \omega^{a}
$$

one finds

$$
\begin{aligned}
\mathbb{A}= & \{\mathbf{i}\}^{T} \ddot{X}+\{\mathbf{b}\}^{T}[\ddot{c}+2 \widetilde{\omega} \dot{c}+\widetilde{\boldsymbol{\omega}}(c+R)+\widetilde{\boldsymbol{\omega}} \widetilde{\boldsymbol{\omega}}(c+R)] \\
& +\{\mathbf{a}\}^{T}\left(\widetilde{\dot{\omega}}^{a}+\widetilde{\omega}^{a} \widetilde{\omega}^{a}\right)\left(R_{c}+\overline{\mathrm{C}}^{T} \bar{\rho}\right) \\
& \left.+\{\mathbf{a}\}^{T}\left[\bar{C}^{T} \ddot{\bar{w}}+2 \widetilde{\omega}^{a} \bar{C}^{T} \dot{\bar{w}}+\left(\widetilde{\dot{\omega}}^{a}+\widetilde{\omega}^{a} \widetilde{\omega}^{a}\right) \bar{C}^{T} \bar{w}\right)\right]
\end{aligned}
$$

where the tilde on a symbol representing a $(3 \times 1)$ matrix indicates the corresponding $(3 \times 3)$ skew-symmetric matrix; for example,

$$
\widetilde{\omega} \triangleq\left[\begin{array}{rrr}
0 & -\omega_{3} & \omega_{2} \\
\omega_{3} & 0 & -\omega_{1} \\
-\omega_{2} & \omega_{1} & 0
\end{array}\right]
$$

Equation (22) calls for the vector $\mathbf{A}$ in the vector basis \{e\}, requiring in Eq. (30) the substitutions from Eqs. (1), (2), and (24)

$$
\begin{aligned}
& \{\mathbf{a}\}^{T}=\{\mathbf{e}\}^{T} \bar{C} \\
& \{\mathbf{b}\}^{T}=\{\mathbf{a}\}^{T} C \underset{{ }_{(2)}}{=}\{\mathbf{e}\}^{T} \bar{C} C \\
& \{\mathbf{i}\}^{T}=\{\mathbf{b}\}^{T} \Theta=\{\mathbf{a}\}^{T} C \Theta=\{\mathbf{e}\}^{T} \bar{C} C \Theta
\end{aligned}
$$

From Eqs. (29) and (31) there follows

$$
\begin{aligned}
\mathbf{A}= & \{\mathbf{e}\}^{T} \bar{A}=\{\mathbf{e}\}^{T}\{\bar{C} C \Theta \ddot{X}+\bar{C} C[\ddot{c}+2 \widetilde{\omega} \dot{c} \\
& +\widetilde{\tilde{\omega}}(c+R)+\widetilde{\omega \omega}(c+R)] \\
& +\bar{C}\left[\widetilde{\dot{\omega}}^{a}\left(R_{c}+\bar{C}^{T} \bar{\rho}\right)+\widetilde{\omega}^{a} \widetilde{\omega}^{a}\left(R_{c}+\bar{C}^{T} \bar{\rho}\right)\right] \\
& \left.+\ddot{\bar{w}}+\bar{C}\left[2 \widetilde{\omega}^{a} \bar{C}^{T} \dot{\bar{w}}+\left(\widetilde{\tilde{\omega}}^{a}+\widetilde{\omega}^{a} \widetilde{\omega}^{a}\right) \overline{\mathrm{C}}^{T} \bar{w}\right]\right\}
\end{aligned}
$$

It should be noted that the quantities $\widetilde{\omega}^{a}$, $\widetilde{\omega}$, and $C$ in Eq. (32) are related by the kinematical equations

$$
\widetilde{\omega}^{\alpha}=\widetilde{\omega}+C \dot{C}^{T}
$$

Using Eq. (11) to remove $\bar{w}$ from $\bar{A}$, and then substituting for $\bar{A}$ from Eq. (32) into Eq. (22), furnishes

$$
\begin{aligned}
\bar{L}= & \int W^{T} D^{T} S D W d v \bar{y}+\int W^{T}\{\bar{C} C \Theta \ddot{X}+\bar{C} C[\ddot{c}+2 \widetilde{\omega} \dot{C}+(\widetilde{\tilde{\omega}}+\widetilde{\omega} \widetilde{\omega})(c+R)] \\
& \left.+\bar{C}\left[\left(\widetilde{\tilde{\omega}}^{a}+\widetilde{\omega}^{a} \widetilde{\omega}^{a}\right)\left(R_{c}+\bar{C}^{T} \bar{\rho}\right)\right]\right\} \mu d v \\
& +\int W^{T} W_{\mu} d v \ddot{\bar{y}}+\int W^{T} \bar{C} 2 \widetilde{\omega}^{a} \bar{C}^{T} W \mu d v \dot{\bar{y}}+\int W^{T} \bar{C}\left(\widetilde{\tilde{\omega}}^{a}+\widetilde{\omega}^{a} \widetilde{\omega}^{a}\right) \bar{C}^{T} W \mu d v \bar{y} \\
& -\int W^{T}\left(\bar{G}-D^{T} \bar{\sigma}_{T}\right) d v+\int W^{T} D^{T} \bar{\sigma}^{\prime} d v
\end{aligned}
$$


The integrals providing the $(6 n \times 6 n)$ matrix coefficients of $\ddot{\bar{y}}, \dot{\bar{y}}$, and $\bar{y}$ are assigned symbols and labels as follows:

$$
\bar{m} \triangleq \int W^{T} W_{\mu} d v, \text { the element consistent mass matrix }
$$

$$
\begin{gathered}
\bar{g} \triangleq 2 \int W^{T} \bar{C} \sim_{\omega^{a}} \bar{C}^{T} W \mu d v, \text { the element gyroscopic } \\
\text { coupling matrix }
\end{gathered}
$$

$\bar{k} \triangleq \int W^{T} D^{T} S D W d v$, the element structural stiffness

$$
\begin{gathered}
\bar{\kappa} \triangleq \int W^{T} \bar{C} \widetilde{\omega}^{a} \widetilde{\omega}^{a} \bar{C}^{T} W \mu d v, \text { the element centrifugal } \\
\text { stiffness matrix }
\end{gathered}
$$

$$
\bar{\alpha} \triangleq \int W^{r} \bar{C} \widetilde{\omega}^{a} \bar{C}^{r} W \mu d v \text {, the element angular acceler- }
$$

ation stiffness matrix

Note that $\bar{m}, \bar{k}$, and $\bar{\kappa}$ are symmetric, while $\bar{g}$ and $\bar{\alpha}$ are skew-symmetric. The bar over these matrices is a reminder that these matrices are associated with the local vector basis $\{\mathbf{e}\}$. When it becomes necessary to consider these matrices as written for the appendage vector basis $\{a\}$, these bars are removed. To obtain $m$ from $\bar{m}$, for example, one may apply a transformation as written below in terms of the $(3 \times 3)$ submatrices $\bar{C}$ and 0 :

$$
[m]=\left[\begin{array}{cccc}
\bar{C}^{T} & 0 & \cdots & 0 \\
0 & \bar{C}^{T} & & \vdots \\
\vdots & \ddots & \bar{C}^{T} & 0 \\
0 & \cdots & 0 & \bar{C}^{T}
\end{array}\right][\bar{m}]\left[\begin{array}{cccc}
\bar{C} & 0 & \cdots & 0 \\
0 & \bar{C} & \vdots \\
\vdots & \ddots & \bar{C} & 0 \\
0 & \cdots & 0 & \bar{C}
\end{array}\right]
$$

and similarly for $k, \kappa, g$, and $\alpha$. The elements of these matrices, such as $m_{i j}$, etc., have indices adopting the $6 \mathrm{n}$ values associated with the six degrees of freedom of each of the $n$ nodal bodies attached to the element in question. (Thus if nodal bodies $11,12,13$, and 14 are attached to element 9 , and nodal body 11 has degrees of freedom $21, \cdots, 26$ associated with $u_{1}^{11}, u_{2}^{11}, u_{3}^{11}, \beta_{1}^{11}, \beta_{2}^{11}, \beta_{3}^{11}$, while nodal body 12 has degrees of freedom $27, \cdots, 32$, etc., the scalar $m_{25,29}^{9}$ is the contribution of the ninth finite element to the 25th row, 29th column of a $6 n \times 6 n$ matrix $M^{c}$ representing the total inertial effect of the $\delta$ finite elements. The role to be played by $M^{c}$, known as the consistent mass matrix, is examined in the penultimate section.)

It may facilitate interpretation to note that the matrices $\bar{C}$ and $\bar{C}^{T}$ in Eqs. (36)-(39) serve merely to transform the matrix lying between them into the local vector basis.

In application to appendages on a spinning base, or to otherwise preloaded structures, the matrix $\bar{k}$ is usually considered in the two parts $\bar{k}_{0}$ and $\bar{k}_{\Delta}$, with elastic stiffness matrix $\bar{k}_{0}$ being the stiffness matrix of the element in its unloaded state, and with the geometric stiffness matrix or preload stiffness matrix $\bar{k}_{\Delta}$ accommodating the influence on stiffness attributed to the preload and often manifested as a consequence of changes in geometry.

Other integrals in Eq. (34) simplify by the removal of terms from the integrand, leaving the matrix $\int W^{p} \mu d v$. Noting that the deformational displacement of the mass center of the sth element is given in the local vector basis by $\bar{w}_{c}^{s}$ in the equation

$$
m_{s} \bar{w}_{c}^{s}=\int_{s} \bar{w} \mu d v=\int_{s} W \mu d v \bar{y}^{s}
$$

where $m_{s}$ is the total mass of the $s$ th finite element, one can define the $\left(3 \times 6 n_{s}\right)$ matrix $W_{c}^{s}$ as the matrix $W^{s}$ evaluated for the element mass center coordinates $\xi_{c}^{s}, \eta_{c}^{s}, \zeta_{c}^{s}$, and write

$$
\int_{s} W_{\mu} d v=m_{s} W_{c}^{s}
$$

Equation (34) can now be rewritten in terms of the notation of Eqs. (35)-(39) and (41), and now, because it will soon become necessary to consider more than one finite 
ellement at a time, the superscript $s$ for the sth element will be added where appropriate, furnishing

$$
\begin{aligned}
& \bar{L}^{s}=\bar{m}^{s} \ddot{\bar{y}}^{s}+\bar{g}^{8} \dot{\bar{y}}^{s}+\left(\bar{k}_{0}^{s}+\bar{k}_{\Delta}^{s}+\bar{\kappa}^{s}+\bar{\alpha}^{s}\right) \bar{y}^{s} \\
& +\int_{s} W^{T} \bar{C}\left(\widetilde{\omega}^{a}+\widetilde{\omega}^{a} \widetilde{\omega}^{a}\right) \bar{C}^{T} \bar{\rho} \mu d v \\
& +\int_{s} \bar{W}^{T} \bar{C} C[\ddot{c}+2 \widetilde{\omega} \dot{c}+(\widetilde{\omega}+\widetilde{\omega} \widetilde{\omega}) c] \mu d v \\
& +m_{s} W_{c}^{s^{T}}\left\{\bar{C}^{s} C \oplus \ddot{X}+\bar{C}^{s} C(\widetilde{\omega}+\widetilde{\omega}) R\right] \\
& \left.+\bar{C}^{s}\left(\widetilde{\tilde{\omega}}^{a}+\widetilde{\omega}^{a} \widetilde{\omega}^{a}\right) R_{c}^{s}\right\} \\
& -\int_{s} W^{T}\left(\bar{G}-D^{T} \overline{\boldsymbol{\sigma}}_{\tau}\right) d v+\int W^{T} D^{T} \overline{\boldsymbol{\sigma}}^{\prime} d v
\end{aligned}
$$

Equation (42) is still not in the desired final form for $\bar{L}^{s}$, because the dependence of $c$ on $\bar{y}^{s}$ has not yet been explicitly accommodated (see Fig. 1 to interpret $-\mathbf{c}=-\{\mathbf{b}\}^{T} \mathbf{c}$ as the displacement of the vehicle mass center $C M$ from its nominal location in $b$ at point $O$ subsequent to steady-state deformation). The mass center shift - $c$ can be attributed in part to the shifts of the mass center locations of the finite elements during deformation, in part to the similar mass center motions of the nodal bodies, and in part to the behavior of moving parts other than the elastic appendage under consideration. If the last of these contributions is simply designated $-e$, and $m$ represents the total vehicle mass, then by mass center definition

$$
\mathbf{c}=\mathbf{e}-\frac{1}{m} \sum_{i=1}^{n} m_{i} \mathbf{u}^{i}-\frac{1}{m} \sum_{r=1}^{\delta} m_{r}\left\{\mathbf{e}^{r}\right\}^{T} \bar{w}_{c}^{r}
$$

for an appendage with $n$ nodes and $\delta$ finite elements. Writing both sides of Eq. (43) in the same basis $\{\mathbf{b}\}$ and substituting from Eq. (40) for $\bar{w}_{c}^{r}$ yields

$$
\{\mathbf{b}\}^{T} c=\{\mathbf{b}\}^{r}\left[e-C^{r}\left(\sum_{i=1}^{n} m_{i} u^{i}+\sum_{r=1}^{\delta} \bar{C}^{r^{T}} \int W \mu d v \bar{y}^{r}\right) / m\right]
$$

which with Eq. (41) becomes (abandoning the unit vectors)

$$
c=e-C^{r}\left(\sum_{i=1}^{n} m_{i} u^{i}+\sum_{r=1}^{\delta} \bar{C}^{r^{T}} m_{r} W_{c}^{r} \bar{y}^{r}\right) / m
$$

Now all terms involving $c$ in Eq. (42) can be removed from the integral over finite element $s$. Rather than differentiate $c$ as it appears in Eq. (45) to obtain $\dot{c}$ and $\ddot{c}$, one can make further use of Eq. (26) and finally obtain $\bar{L}^{s}$ from Eq. (42) in the form

$$
\begin{aligned}
& \bar{L}^{s}=\bar{m}^{s} \ddot{\bar{y}}^{s}-m_{s} W_{c}^{s^{T}} \bar{C}^{s^{T}}\left(\sum_{r=1}^{\delta} \bar{C}^{r^{r}}\left(m_{r} W_{c}^{r} \ddot{\bar{y}}^{r}+\sum_{i=1}^{n} m_{i} \ddot{u}^{i}\right) / O m\right. \\
& +\bar{g}^{s} \dot{\bar{y}}^{s}-2 m m_{s} W_{c}^{s^{T}} \bar{C}^{s^{T}} \widetilde{\omega}^{a}\left(\sum_{r=1}^{\delta} \bar{C}^{r^{T}} m_{r} W_{c}^{r} \dot{\bar{y}}^{r}+\sum_{i=1}^{n} m_{i} \dot{u}^{i}\right) / m \\
& +\left(\bar{k}_{0}^{s}+\bar{k}_{\Delta}^{s}+\bar{\kappa}^{s}+\bar{\alpha}^{s}\right) \bar{y}^{s} \\
& -m_{s} W_{c}^{s^{T}} \bar{C}^{s^{T}}\left(\widetilde{\tilde{\omega}}^{a}+\widetilde{\omega}^{a} \widetilde{\omega}^{a}\right) \cdot\left(\sum_{r=1}^{\delta} \bar{C}^{r T} m_{r} W_{c}^{r} \bar{y}^{r}+\sum_{i=1}^{n} m_{i} u^{i}\right) / m \\
& +\int_{s} W^{T} \bar{C}\left(\widetilde{\tilde{\omega}}^{a}+\widetilde{\omega^{a}} \widetilde{\omega}^{a}\right) \bar{C}^{T} \bar{\rho} \mu d v \\
& +m_{s} W_{c}^{s^{p}}\left\{\bar{C}^{s} C[\Theta \ddot{X}+(\widetilde{\hat{\omega}}+\widetilde{\omega} \widetilde{\omega}) R]+\bar{C}^{s}\left(\widetilde{\tilde{\omega}}^{a}+\widetilde{\omega}^{a} \widetilde{\omega}^{a}\right) R_{c}^{s}\right\} \\
& -\int_{s} W^{T}\left(\bar{G}-D^{T} \overline{\boldsymbol{\sigma}}_{\tau}\right) d v+\int_{s} W^{T} D^{T} \overline{\boldsymbol{\sigma}}^{\prime} d v \\
& +m_{\mathrm{c}}^{s} W_{\mathrm{c}}^{s^{T}} \bar{C}^{s} C[\ddot{e}+2 \widetilde{\omega} \dot{e}+(\widetilde{\tilde{\omega}}+\widetilde{\omega} \tilde{\omega}) e]
\end{aligned}
$$


Equation (46), repeated $\delta$ times for elements $s=1, \cdots, \delta$, provides in the matrices $\bar{L}^{1}, \cdots, \bar{L}^{\bar{\delta}}$ a representation of the contribution of structural interactions to the forces $\mathbb{F}^{1}, \cdots, \mathbb{F}^{n}$ and the torques $\mathbf{T}^{1}, \cdots, \mathbf{T}^{n}$ applied to the $n$ nodal bodies. There remains the task of deriving equations of motion of these nodal bodies.

\section{Nodal Body Equations of Motion}

For the $j$ th nodal body, having mass $m_{j}$ and inertial acceleration $\mathbf{A}^{j}$, the translation equation

$$
\mathbb{F}^{j}=m_{j} \mathbf{A}^{j}
$$

can be expressed in the desired form by inspection of the results for a generic point of a finite element. The acceleration $\mathbf{A}^{j}$ is defined in terms of the symbols of Fig. 1 as

$$
\mathbf{A}^{j} \triangleq \frac{i}{=} \frac{d^{2}}{d t^{2}}\left(\mathbf{X}+\mathbf{c}+\mathbf{R}+\mathbf{r}^{j}+\mathbf{u}^{j}\right)
$$

which can be compared to Eq. (23) for the element field point. A line of argument parallel to that providing Eq. (29) from Eq. (23) produces from Eq. (48) the expression

$$
\mathbf{A}^{j}=\{\mathbf{i}\}^{T} \ddot{X}+\{\mathbf{b}\}^{T}[\ddot{c}+2 \widetilde{\omega} \dot{c}+\widetilde{\omega}(c+R)+\widetilde{\omega}(c+R)]+\{\mathbf{a}\}^{T}\left[\left(\widetilde{\omega}^{a}+\widetilde{\omega}^{a} \widetilde{\omega}^{a}\right)\left(r^{j}+u^{j}\right)+2 \widetilde{\omega}^{a} \dot{u}^{j}+\ddot{u}^{j}\right]
$$

The matrix $c$ can be substituted from Eq. (45), and by the argument leading from there to Eq. (46) one can develop from Eqs. (47) and (49) (with appropriate change of vector basis)

$$
\begin{aligned}
& \mathbf{F}^{j}=\{\mathbf{a}\}^{T} F^{j}=\{\mathbf{a}\}^{T} m_{j}\{C \oplus \ddot{X}+C[\ddot{e}+2 \tilde{\omega} \dot{e}+\widetilde{\boldsymbol{\omega}}(e+R) \\
& +\widetilde{\omega \omega}(e+R)]+\left(\widetilde{\tilde{\omega}^{a}}+\widetilde{\omega}^{a} \widetilde{\omega}^{a}\right) r^{j}+\ddot{u}^{j} \\
& -\left(\sum_{i=1}^{n} m_{i} \ddot{u}_{i}+\sum_{r=1}^{\delta} \bar{C}^{r^{r}}\left(m_{r} W_{c}^{r} \ddot{\bar{y}}^{r}\right) / m\right. \\
& +2 \widetilde{\omega}^{a}\left[\dot{u}^{j}-\left(\sum_{i=1}^{n} m^{i} \dot{u}^{i}+\sum_{r=1}^{\delta} \overline{\mathrm{C}}^{r} m_{r} W_{c}^{r} \dot{\bar{y}}^{r}\right) / m\right] \\
& \left.+\left(\tilde{\tilde{\omega}}^{a}+\widetilde{\omega}^{a} \widetilde{\omega}^{a}\right)\left[u^{j}-\left(\sum_{i=1}^{n} m^{i} u^{i}+\sum_{r=1}^{\delta} \bar{C}^{r}{ }^{r} m_{r} W_{c}^{r} \bar{y}^{r}\right) / m\right]\right\}
\end{aligned}
$$

The force $\mathbf{F}^{j}$ applied to the $j$ th nodal body consists of the external force $\mathbf{f}^{j}=\{\mathbf{a}\}^{T} f^{j}$ applied at that node plus the structural interaction forces $F^{s j}$ applied to node $j$ by adjacent structural elements $s$. If the symbol $\sum_{s \in \varepsilon_{j}}$ denotes summation over those values of $s$ belonging to the set $\delta_{j}$ consisting of that subset of the element numbers $1, \cdots, \delta$ corresponding to elements in contact with node $j$, then $\mathrm{F}^{j}$ becomes

$$
\mathbf{F}^{j}=\mathbf{f}^{j}+\sum_{s \in \delta} \mathbf{F}^{s j}
$$

If $\mathbf{F}^{s j}$ is written in the vector basis $\left\{\mathbf{e}^{s}\right\}$ as

$$
\mathbf{F}^{s j} \triangleq\left\{\mathbf{e}^{s}\right\}^{T} \bar{F}_{(2)}^{s j}=\{\mathbf{a}\}^{T} \bar{C}^{s^{T}} \bar{F}^{s j}
$$


and the relationship $\bar{F}^{s j}=-\bar{F}^{j s}$ is accepted as a consequence of Newton's third law, one can extract from Eq. (50) the matrix equations

$$
\begin{aligned}
& f^{j}-\sum_{s \in \tilde{\delta}_{j}} \bar{C}^{s^{T}} \bar{F}^{j s}=m^{j}\{C \otimes \ddot{X}+C[\ddot{e}+2 \widetilde{\omega} \dot{e}+(\widetilde{\omega}+\widetilde{\omega} \widetilde{\omega})(e+R)] \\
& +\left(\widetilde{\tilde{\omega}}^{a}+\widetilde{\omega}^{a} \widetilde{\omega}^{a}\right) r^{j}+\ddot{u}^{j}-\left(\sum_{i=1}^{n} m^{i} \ddot{u}^{i}+\sum_{r=1}^{\delta} \bar{C}^{r^{r}} m_{r} W_{c}^{r} \ddot{\bar{y}}^{r}\right) / m \\
& +2 \widetilde{\omega}^{a}\left[\dot{u}^{j}-\left(\sum_{i=1}^{n} m^{i} \dot{u}^{i}+\sum_{r=1}^{\delta} \bar{C}^{r^{r}}\left(m_{r} W_{c}^{r} \dot{\bar{y}}^{r}\right) / m\right]\right. \\
& \left.+\left(\widetilde{\omega}^{a}+\widetilde{\omega}^{a} \widetilde{\omega}^{a}\right)\left[u^{j}-\left(\sum_{i=1}^{n} m^{i} u^{i}+\sum_{r=1}^{\delta} \bar{C}^{r} m_{r} W_{c}^{r} \bar{y}^{r}\right) / m\right]\right\} \\
& j=1, \cdots, n
\end{aligned}
$$

Here for convenience in future composition of matrix equations the $(3 \times 3)$ unit matrix $U$ has been used to define the mass matrix

$$
m^{j}=m_{j} U
$$

By systematically examining the quantities $\bar{L}$ defined in Eq. (19) and appearing in the $\delta$ matrix equations represented by Eq. (46), one can extract expressions for the quantities $\bar{F}^{j s}$ appearing in Eq. (53); upon substitution of these expressions, one has in Eq. (53) a set of dynamical equations in $u^{j}$ and $\bar{y}^{s}, j=1, \cdots, n, s=1, \cdots, \mathcal{E}$. By the definition found after Eq. (9), the matrices $\bar{y}^{1}, \cdots, \bar{y}^{\varepsilon}$ are comprised of the matrices $\bar{u}^{1}, \cdots, \bar{u}^{n}, \bar{\beta}^{1}, \cdots, \bar{\beta}^{n}$, which transform to $u^{i}$ and $\beta^{i}$ by $\bar{u}^{i}=\bar{C} u^{i}$ and $\bar{\beta}^{i}=\bar{C} \beta^{i}$, $i=1, \cdots, n$. Thus Eq. (53), with substitutions from Eq. (46), provides $3 n$ scalar second-order differential equations in the $6 n$ unknowns $u_{\alpha^{2}}^{1} \cdots, u_{\alpha^{3}}^{n} \beta_{\alpha^{3}}^{1}, \cdots, \beta_{\alpha^{\prime}}^{n}$ $\alpha=1,2,3$. Completion of the set requires the equations of rotational motion of the nodal bodies.

The basic equation for the rotation of the $j$ th nodal rigid body is

$$
\mathbf{T}^{j}=\dot{\mathbf{H}}^{j}=\boldsymbol{1}^{j} \cdot \dot{\boldsymbol{\omega}}^{j}+\dot{\mathbf{j}}^{j} \cdot \boldsymbol{\omega}^{j}=\boldsymbol{\|}^{j} \cdot \dot{\boldsymbol{\omega}}^{j}+\boldsymbol{\omega}^{j} \times \boldsymbol{\|}^{j} \cdot \boldsymbol{\omega}^{j}
$$

where $\boldsymbol{T}^{j}$ is the applied torque, $\mathbf{H}^{j}$ the angular momentum, and $\|^{j}$ the inertia dyadic of the nodal body, all referred to the mass center of the body, and over-dot denotes time differentiation in an inertial frame of reference. The inertial angular velocity $\omega^{j}$ of the $j$ th body may be expressed in terms of established notation as

$$
\omega^{j}=\omega^{a}+\{\mathbf{a}\}^{T} \dot{\beta}^{j}=\{\mathbf{a}\}^{T}\left(\omega^{a}+\dot{\beta}^{j}\right)
$$

and its inertial derivative is

$$
\dot{\boldsymbol{\omega}}^{j}=\dot{\boldsymbol{\omega}}^{a}+\{\mathbf{a}\}^{T} \ddot{\beta}^{j}+\boldsymbol{\omega}^{a} \times\{\mathbf{a}\}^{T} \dot{\beta}^{j}=\{\mathbf{a}\}^{T}\left(\dot{\omega}^{a}+\ddot{\beta}^{j}+\widetilde{\omega}^{a} \dot{\beta}^{j}\right)
$$

so that Eq. (55) becomes

$$
\begin{aligned}
\mathbf{T}^{j}=\{\mathbf{a}\}^{T} T^{j}= & \left\{\mathbf{n}^{j}\right\}^{T} I^{j}\left\{\mathbf{n}^{j}\right\} \cdot\{\mathbf{a}\}^{T}\left(\dot{\omega}^{a}+\ddot{\beta}^{j}+\widetilde{\omega}^{a} \dot{\beta}^{j}\right) \\
& +\{\mathbf{a}\}^{T}\left(\omega^{a}+\dot{\beta}^{j}\right) \times\left\{\mathbf{n}^{j}\right\}^{T} I^{j}\left\{\mathbf{n}^{j}\right\} \cdot\{\mathbf{a}\}^{T}\left(\omega^{a}+\dot{\beta}^{j}\right)
\end{aligned}
$$


where $\left\{\mathbf{n}^{j}\right\}$ is the $(3 \times 1)$ array of dextral, orthogonal unit vectors $\mathbf{n}_{1}^{j}, \mathbf{n}_{2}^{j}, \mathbf{n}_{3}^{j}$, fixed in nodal body $j$, and coincident with (a) when the appendage is in its steady state (see Fig. 1). The direction cosine matrix relating $\left\{\mathbf{n}^{j}\right\}$ and $\{\mathbf{a}\}$ subsequent to small appendage deformation is given by the relationship

$$
\left\{\mathbf{n}^{j}\right\}=\left(U-\widetilde{\beta}^{j}\right)\{\mathbf{a}\}
$$

where $U$ is the $(3 \times 3)$ unit matrix and $\widetilde{\beta}^{j}$ is the skewsymmetric matrix formed of the elements $\beta_{1}^{j}, \beta_{2}^{j}, \beta_{3}^{j}$ according to the pattern of Eq. (30); i.e.,

$$
\tilde{\beta}_{\alpha \theta}^{j} \triangleq \epsilon_{\alpha \gamma \theta} \beta_{\gamma}^{j}
$$

where $\epsilon_{\alpha \gamma_{\theta}}$ is the epsilon symbol of tensor analysis.

Substituting Eq. (59) into Eq. (58) produces a vector equation entirely in the $\{\mathbf{a}\}$ basis, or equivalently the matrix equation

$$
\begin{aligned}
T^{j}= & I^{j} \dot{\omega}^{a}+\widetilde{\omega}^{a} I^{j} \omega^{a}+I^{j} \ddot{\beta}^{j}+\left[\widetilde{\omega}^{a} I^{j}-\left(I^{j} \omega^{a}\right)^{\sim}+I^{j} \widetilde{\omega}^{a}\right] \dot{\beta}^{j} \\
& +\left[I^{j} \widetilde{\dot{\omega}}^{a}-\left(I^{j} \dot{\omega}^{a}\right)^{\sim}+\widetilde{\omega}^{a} I^{j} \widetilde{\omega}^{a}-\widetilde{\omega}^{a}\left(I^{j} \omega^{a}\right)^{\sim}\right] \beta^{j}
\end{aligned}
$$

where second-degree terms in the matrix $\beta^{j}$ and its derivatives have been ignored, and the tilde retains its operational significance (see Eq. 30), so that, for example,

$$
\left(I^{j} \omega^{\alpha}\right)_{\alpha \theta} \triangleq \epsilon_{\alpha \gamma_{\theta}} I_{\gamma \eta}^{j} \omega_{\eta}^{a}
$$

The torque $\mathbf{T}^{j}$ applied to the $j$ th nodal body consists of the external torque $\mathbf{t}^{j}=\{\mathrm{a}\}^{T} t^{j}$ applied at that node plus the structural interaction torques $\mathrm{T}^{s j}$ applied to node $j$ by adjacent structural elements $s$. If, as in Eq. (51), the set $\delta_{j}$ contains the numbers of the elements in contact with node $j$, then $\mathbf{T}^{j}$ may be written (in parallel with Eqs. 51 and 52 ) as

$$
\begin{aligned}
\mathbf{T}^{j}=\{\mathbf{a}\}^{T} \mathbf{T}^{j} & =\{\mathbf{a}\}^{T} t^{j}+\sum_{s \in \mathscr{E}_{j}} \mathbf{T}^{s j}=\{\mathbf{a}\}^{T} t^{j}-\sum_{s \in \mathscr{S}_{j}} \mathbb{T}^{j s} \\
& =\{\mathbf{a}\}^{T}\left[t^{j}-\sum_{s \in \mathcal{E}_{j}} \overline{\boldsymbol{C}}^{s^{T}} \overline{\mathbf{T}}^{j s}\right]
\end{aligned}
$$

The combination of Eqs. (60) and (61) provides

$$
\begin{aligned}
t^{j}-\sum_{s \in \tilde{\omega}_{j}} \bar{C}^{s^{T}} \bar{T}^{j s}= & I^{j} \dot{\omega}^{a}+\widetilde{\omega}^{a} I^{j} \omega^{a}+I^{j} \dddot{\beta}^{j} \\
& +\left[\widetilde{\omega}^{a} I^{j}-\left(I^{j} \omega^{a}\right)^{\sim}+I^{j} \widetilde{\omega}^{a}\right] \dot{\beta}^{j} \\
& +\left[I^{j} \widetilde{\omega}^{a}-\left(I^{j} \dot{\omega}^{a}\right)^{\sim}+\widetilde{\omega}^{a} I^{j} \widetilde{\omega}^{a}-\widetilde{\omega}^{a}\left(I^{j} \omega^{a}\right)^{\sim}\right] \beta^{j} \\
& \quad j=1, \cdots, n
\end{aligned}
$$

The rotational Eqs. (62) stand in parallel with the translational Eqs. (53) as the basic equations of motion of the $n$ nodal bodies of the appendage. Once Eqs. (46) and (19) have been used to provide expressions for the matrices $\bar{T}^{j s}$ and $\bar{F}^{j s}$ appearing respectively in Eqs. (62) and (53), these constitute a complete set of dynamical equations.

\section{Coordinate Transformations}

There remains the critical task of packaging Eqs. (53) and (62), with substitutions from Eq. (46), in a form convenient for the generation of coordinate transformations. To this end, let

$$
q \triangleq\left(u_{1}^{1} u_{2}^{1} u_{3}^{1} \beta_{1}^{1} \beta_{2}^{1} \beta_{3}^{1} u_{1}^{2}, \cdots, \beta_{3}^{n}\right)^{n}
$$

be the $(6 n \times 1)$ matrix of nodal deformation coordinates, and rewrite the $6 n$ second-order differential equations implied by Eqs. (46), (53), and (62) in the form

$$
M^{\prime} \ddot{q}+D^{\prime} \dot{q}+G^{\prime} \dot{q}+K^{\prime} q+A^{\prime} q=L^{\prime}
$$

where $M^{\prime}, D^{\prime}$, and $K^{\prime}$ are $(6 n \times 6 n)$ symmetric matrices and where $G^{\prime}$ and $A^{\prime}$ are $(6 n \times 6 n)$ skew-symmetric matrices, with $L^{\prime}$ a $(6 n \times 1)$ matrix not involving the deformation variables in $q$. Since Eqs. (53), (62), and (46) are all linear in the variables $u^{j}, \beta^{j}$, and $\bar{y}^{j}$ contained within $q$, and since any square matrix can be written as the sum of symmetric and skew-symmetric parts, the possibility of expression of these equations in the form of Eq. (64) is guaranteed by the symmetric character of the coefficients of $\ddot{u}^{j}, \ddot{\beta}^{j}$, and $\ddot{\bar{y}}^{j}$ in the constituent equations. 
The $(6 n \times 6 n)$ matrix $M^{\prime}$ can be represented as the sum of three parts, as symbolized by

$$
M^{\prime} \triangleq M+M^{c}+\bar{M}
$$

where $M$ is null except for the $(3 \times 3)$ matrices $m^{1}, I^{1}$, $m^{2}, \cdots, I^{n}$ along its principal diagonal, $M^{c}$ is the consistent mass matrix whose elements $M_{i j}^{c}$ are given in terms of the constituents of the finite element inertia matrices $m$ in Eq. (40) by

$$
M_{i j}^{c}=\sum_{s=1}^{\mathcal{E}} m_{i j}^{s}
$$

and the contribution $\bar{M}$ accommodates the reduction of the effective inertia matrix due to mass center shifts within the vehicle induced by deformation (see for example the terms

$$
-\left(\sum_{i=1}^{n} m^{i} \ddot{u}^{i}+\sum_{r=1}^{\varepsilon} \bar{C}^{r^{T}} M_{r} W_{c}^{r} \ddot{\bar{y}}^{r}\right) / m
$$

in Eq. 53).

The matrix $D^{\prime}$ in Eq. (64) accommodates any viscous damping that may be introduced to represent energy dissipation due to structural vibrations. As Eqs. (62), (53), and (46) have been formulated here, such terms have been omitted, but they can still be inserted if one accepts the practice common among structural dynamicists of incorporating the equivalent of a term $D^{\prime} \dot{q}$ into equations of vibration only after derivation of equations of motion and transformation of coordinates.

Examination of the coefficients of $\dot{\beta}^{j}, \dot{u}^{j}$, and $\dot{\bar{y}}^{j}$ in Eqs. (62), (53), and (46) reveals that all have coefficients which will appear in the skew-symmetric matrix $G^{\prime}$ in Eq. $(64)^{2}$; since all such terms disappear when $\omega^{a}$ is nominally zero, the matrix $G^{\prime}$ is said to provide the gyroscopic coupling of the equations of vibration. Note that the ma- trices $\bar{g}^{8}$ defined generically in Eq. (37) contribute to $G^{\prime}$ just as the matrices $\bar{m}^{s}$ contribute to $M^{\prime}$ (see Eqs. 65 and 66).

The terms from Eqs. (62), (53), and (46) contributing to the matrix $K^{\prime}$ in Eq. (64) are basically of three kinds: (1) those represented by $\bar{k}_{0}^{s}$ in Eq. (46), which reflect the elastic stiffness of the structure in its unloaded state, (2) those represented by $\bar{k}_{\Delta}^{s}$ in Eq. (46), which provide the increment to the elastic stiffness of the structure attributable to structural preload, and (3) those represented in Eq. (46) by $\bar{\kappa}^{s}$ and in Eqs. (46), (53), and (62) by other terms involving base acceleration (such as the centripetal acceleration term $m^{j} \widetilde{\omega^{a}} \widetilde{\omega}^{a}$ in Eq. 53). The elements of the matrices $\bar{k}_{0}^{s}, \bar{k}_{\Delta^{s}}^{s}$ and $\bar{\kappa}^{s}$ contribute to $K^{\prime}$ in a manner analogous to the contribution of $\bar{m}^{s}$ to $M^{\prime}$ (see Eqs. 65 and 66 ).

Finally, the matrix $A^{\prime}$ in Eq. (64) contains all terms from Eqs. (46), (53), and (62) involving $\dot{\omega}^{a}$, and in addition the coefficient $-\widetilde{\omega}^{a}\left(I^{j} \omega^{a}\right)^{\sim}$ of $\beta^{j}$ in Eq. (62) makes a contribution to $A^{\prime}$. Because certain of the coordinate transformation procedures to be considered depend upon the absence of the matrix $A^{\prime}$, it is worthwhile to examine the skewsymmetric part of the matrix $-\widetilde{\omega}^{a}\left(I^{j} \omega^{a}\right)^{\sim}$ in detail, since when $\omega^{a}$ has some nominal constant value, say $\Omega$, and $\dot{\omega}^{a}$ is nominally zero, this matrix is the sole contributor to $A^{\prime}$. In terms of its symmetric and skew-symmetric parts, this matrix is

$$
\begin{aligned}
-\widetilde{\omega}^{a}\left(I^{j} \omega^{a}\right)^{\sim}= & -\frac{1}{2}\left[\widetilde{\omega}^{a}\left(I^{j} \omega^{a}\right)^{\sim}+\left(I^{j} \omega^{a}\right)^{\sim} \widetilde{\omega}^{a}\right] \\
& -\frac{1}{2}\left[\widetilde{\omega}^{a}\left(I^{j} \omega^{a}\right)^{\sim}-\left(I^{j} \omega^{a}\right)^{\sim} \widetilde{\omega}^{a}\right]
\end{aligned}
$$

The matrix identity

$$
\tilde{x} \tilde{y}-\tilde{y} \tilde{x}=(\tilde{x} y)^{\sim}
$$

for any $(3 \times 1)$ matrices $x$ and $y$ permits the skew-symmetric part of $-\widetilde{\omega}^{a}\left(I^{j} \omega^{a}\right)^{\sim}$ to be recorded as

$$
-\frac{1}{2}\left[\widetilde{\omega}^{a}\left(I^{j} \omega^{a}\right)^{\sim}-\left(I^{j} \omega^{a}\right)^{\sim} \widetilde{\omega}^{a}\right]=-\frac{1}{2}\left[\widetilde{\omega}^{a} I^{j} \omega^{a}\right]^{\sim} \approx-\frac{1}{2}\left[\widetilde{\Omega} I^{j} \Omega\right]^{\sim}
$$

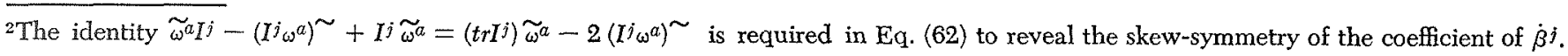


where the final substitution replaces $\omega^{a}$ by its nominal value, $\Omega$. In terms of scalars representing the elements $I_{\alpha \theta}$ of $I^{j}$ and $\Omega_{\theta}$ of $\Omega$, the independent nonzero terms of $-(1 / 2)\left[\widetilde{\Omega} I^{j} \Omega\right]^{\sim}$ are given by

$$
\begin{aligned}
-\frac{1}{2}\left[\widetilde{\Omega} I^{j} \Omega\right]_{12}^{\sim}= & -\frac{1}{2}\left[\left(I_{11}-I_{22}\right) \Omega_{1} \Omega_{2}+I_{12}\left(\Omega_{2}^{2}-\Omega_{1}^{2}\right)\right. \\
& \left.+I_{13} \Omega_{2} \Omega_{3}-I_{23} \Omega_{1} \Omega_{3}\right] \\
-\frac{1}{2}\left[\widetilde{\Omega} I^{j} \Omega\right]_{13}^{\sim}= & -\frac{1}{2}\left[\left(I_{11}-I_{33}\right) \Omega_{1} \Omega_{3}+I_{13}\left(\Omega_{3}^{2}-\Omega_{1}^{2}\right)\right. \\
& \left.+I_{12} \Omega_{2} \Omega_{3}-I_{32} \Omega_{1} \Omega_{2}\right]
\end{aligned}
$$

and

$$
\begin{aligned}
-\frac{1}{2}\left[\widetilde{\Omega} I^{j} \Omega\right]_{23}^{\sim}= & -\frac{1}{2}\left[\left(I_{22}-I_{33}\right) \Omega_{2} \Omega_{3}+I_{23}\left(\Omega_{3}^{2}-\Omega_{2}^{2}\right)\right. \\
& \left.+I_{21} \Omega_{1} \Omega_{3}-I_{31} \Omega_{1} \Omega_{2}\right]
\end{aligned}
$$

Since such terms as these are the sole contributors to $A^{\prime}$ when $\dot{\omega}^{a}$ is nominally zero, it becomes clear that the special case $A^{\prime}=0$ applies when the base experiences small excursions about a nonzero constant spin only if the nodal bodies are particles or spheres (or in the extraordinary case when, in the steady-state of deformation, all nodal bodies have principal axes of inertia aligned with the nominal value of the angular velocity $\omega^{a}$.)

The objective of this section is to find a coordinate transformation which will permit the replacement of the homogeneous form of Eq. (64) with a set of completely uncoupled differential equations. Since the conceptual, analytical, and computational difficulties encountered in meeting this objective in general terms are greatly diminished in special cases of practical interest, consideration will be given both to the general case of Eq. (64) and to a variety of simpler restricted forms of Eq. (64) for which alternative coordinate transformations may be found.

Inspection of Eqs. (62), (53), and (46) reveals that the coefficients of $q$ and $\dot{q}$ in Eq. (64) depend upon $\omega^{a}$, which characterizes the rotational motion of the appendage base. For the problems of interest, $\omega^{a}$ is an unknown function of time, to be determined only after the appendage Eqs. (64) are augmented by other equations of dynamics and control for the total vehicle and solved. Only if $\omega^{a}$ can be assumed to experience, in a given time interval, small excursions about a constant nominal value (say $\Omega$ ) is there any possibility of obtaining from Eq. (64) a transformation to uncoupled equations. Any methods involving modal coordinates (see Section I) depend formally upon this assumption, which is adopted henceforth. With this restriction, the coefficient matrices of $q, \dot{q}$, and $\ddot{q}$ in Eq. (64) are constants, since products of small quantities are to be ignored. In practice, this restriction is negotiable.

If all of the matrices $A^{\prime}, K^{\prime}, G^{\prime}, D^{\prime}, M^{\prime}$ and $L^{\prime}$ in Eq. (64) are constant but nonzero, there exists no transformation of the form $q=\phi \eta$, with $\eta$ a $(6 n \times 1)$ matrix of new coordinates, which can be used to obtain from Eq. (64) a secondorder differential equation in $\eta$ with diagonal coefficient matrices. In order to transform Eq. (64) to a set of uncoupled equations it is first necessary to rewrite Eq. (64) in first-order form, such as

$$
\mathscr{A}+B Q=\mathscr{P}
$$

where

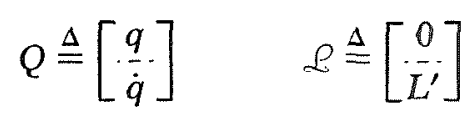

$$
A \triangleq\left[\begin{array}{c:c:c}
K^{\prime}+A^{\prime} & 0 \\
\hdashline 0 & M^{\prime}
\end{array}\right] \quad B \triangleq\left[\begin{array}{c:c}
0 & -K^{\prime}-A^{\prime} \\
\hdashline K^{\prime}+A^{\prime} & D^{\prime}+G^{\prime}
\end{array}\right]
$$

Now let $\Phi$ be a $(12 n \times 12 n)$ matrix of (complex) eigenvectors of the differential operator in $\mathbb{E q}$. (71), and let $\Phi^{\prime}$ be a $(12 n \times 12 n)$ matrix of (complex) eigenvectors of the homogeneous adjoint equation

$$
\mathscr{A}^{T} \dot{Q}^{\prime}+B^{r} Q^{\prime}=0
$$

so that $\Phi$ and $\Phi^{\prime}$ are related by (Ref. 16)

$$
\Phi^{-1}=\ell \Phi^{\prime T}
$$

with $\ell$ a $(12 n \times 12 n)$ diagonal matrix which depends upon the normalization of $\Phi$ and $\Phi^{\prime}$. Substitution into Eq. (64) of the transformation

$$
Q=\Phi Y
$$

and premultiplication by $\Phi^{\prime \prime}$ furnishes

$$
\left(\Phi^{\prime T} \Re \Phi\right) \dot{Y}+\left(\Phi^{\prime T} \bigotimes \Phi\right) Y=\Phi^{\prime T} \mathscr{L}
$$

The two coefficient matrices enclosed in parentheses are diagonal (as is evident from Eq. 73 when $\mathscr{A}=U$, which by virtue of the nonsingularity of $A$ can be assumed for this proof without loss of generality). If $\Lambda$ is the $(12 n \times 12 n)$ matrix of the (complex) eigenvalues of the differential operator in Eq. (71) (or Eq. 72, which has the same eigen- 
values), then upon premultiplication by $\left(\Phi^{\prime T}(A \Phi)^{-1}\right.$ one obtains

$$
\dot{Y}=\Lambda Y+\left(\Phi^{\prime T} \mathscr{A} \Phi\right)^{-1} \Phi^{\prime T} \mathscr{L}
$$

which is in a form convenient for computation. (Note that the matrix inversion in Eq. (76) consists simply of calculating the reciprocals of the diagonal elements of $\Phi^{\prime T} \mathscr{A} \Phi$.) In practice, one may expect that physical interpretation of the new (complex) state variables $Y_{1}, \cdots, Y_{12 n}$ (see Ref. 8) will permit truncation to a reduced set of variables contained in a new $(2 N \times 1)$ matrix $Y$, and with corresponding truncation of $\Lambda$ to the $(2 N \times 2 N)$ matrix $\bar{\Lambda}$ and truncation of $\Phi$ and $\Phi^{\prime}$ to the $(12 n \times 2 N)$ matrices $\bar{\Phi}$ and $\bar{\Phi}^{\prime}$, one can reduce $\mathrm{Eq} .(76)$ to

$$
\dot{\bar{Y}}=\overline{\Lambda Y}+\left(\bar{\Phi}^{\prime T} \mathscr{A} \bar{\Phi}\right)^{-1} \bar{\Phi}^{\prime T} \mathscr{L}
$$

Equation (77) may be used in conjunction with vehicle equations of motion to simulate system behavior.

In the special case for which $A^{\prime}=D^{\prime}=0$, the matrices $A$ and $\Leftrightarrow$ in $\mathrm{Eq}$. (71) are respectively symmetric and skew symmetric, so that Eq. (72) becomes

$$
\mathscr{A} \dot{Q}^{\prime}-B Q^{\prime}=0
$$

and the adjoint eigenvector matrix is available as the complex conjugate ${ }^{3}$

$$
\Phi^{\prime}=\Phi^{*}
$$

After truncation, Eq. (79) can be substituted into Eq. (77), so that in this special case the final equations are obtained without the necessity of actually computing the eigenvectors constituting $\Phi^{\prime}$.

Construction of Eq. (77) requires, in general, however, the computation from $A$ and $B$ of the $2 N$ eigenvalues in the matrix $\bar{\Lambda}$ and the corresponding eigenvectors in the two matrices $\bar{\Phi}$ and $\bar{\Phi}^{\prime}$. It is always possible to avoid the task of computing the adjoint equation eigenvectors in $\bar{\Phi}^{\prime}$, but only at the cost of a matrix inversion and some sacrifice in the rigor of the procedure. Having decided as previously upon the relevant modes of appendage deformation and constructed the $6 n \times 2 N$ matrix $\bar{\Phi}$, one can rewrite Eq. (71) in the form

$$
\dot{Q}=B Q+L
$$

where

$$
B \triangleq\left[\begin{array}{c:c}
0 & U \\
\hdashline-M^{\prime-1}\left(K^{\prime}+A^{\prime}\right) & -M^{\prime-1}\left(D^{\prime}+G^{\prime}\right)
\end{array}\right] \text { and } L \triangleq\left[\begin{array}{c}
0 \\
\hdashline M^{\prime-1} L^{\prime}
\end{array}\right]
$$

and introduce the transformation

$$
Q=\bar{\Phi} \bar{Y}
$$

recognizing the constraint that this substitution imposes upon the solution, and then premultiply by the pseudoinverse (Ref. 17)

$$
(\bar{\Phi})^{\dagger}=\left(\bar{\Phi}^{T} \bar{\Phi}\right)^{-1} \bar{\Phi}^{T}
$$

to obtain

$$
\dot{\bar{Y}}=\bar{\Phi}^{\dagger} B \bar{\Phi} \bar{Y}+\bar{\Phi}^{\dagger} L
$$

Since $\Lambda$ and $\Phi$ contain respectively the eigenvalues and eigenvectors of $B$, one can write

$$
B \Phi=\Phi \Lambda
$$

If now $\Phi$ and $\Lambda$ are written in partitioned form as

$$
\Phi \triangleq\left[\begin{array}{l:l}
\Phi & \bar{\Phi}
\end{array}\right] \quad \Lambda \triangleq\left[\begin{array}{c:c}
\bar{\Lambda} & 0 \\
\hdashline 0 & \overline{\bar{\Lambda}}
\end{array}\right]
$$

then Eq. (84) becomes

$$
[B \bar{\Phi}: B \overline{\bar{\Phi}}]=[\bar{\Phi} \bar{\Lambda} ; \overline{\bar{\Phi}} \overline{\bar{\Lambda}}]
$$

The equality of the matrices in the first partitions leads to

$$
\bar{\Phi}^{\dagger} B \bar{\Phi}=\bar{\Lambda}
$$

which when substituted with Eq. (82) into Eq. (83) yields

$$
\dot{\bar{Y}}=\bar{\Lambda} \bar{Y}+\left(\bar{\Phi}^{T} \bar{\Phi}\right)^{-1} \bar{\Phi}^{T} L
$$

${ }^{3}$ This observation is a contribution of Mr. A. S. Hopkins of UCLA and McDonnell-Douglas Corp. 
as an alternative to $\mathrm{Eq} .(77){ }^{4}$

Substitution of Eq. (77) or Eq. (88) for Eq. (64) introduces conceptual and computational problems that can be avoided if in Eq. (64) the matrix $A^{\prime}=0$ and if in addition one of the three following restrictions is applicable: (1) $G^{\prime}=0$ (see Ref. 18 for details); (2) $G^{\prime}=0$ and $D^{\prime}$ is a polynomial in $M^{\prime}$ and $K^{\prime}$ (see Ref. 19); or (3) $D^{\prime}=0$ (see Ref. 20 for the underlying theory).

Only the last of these special cases requires elaboration beyond published references. In this case the homogeneous form of Eq. (64) becomes

$$
M^{\prime} \ddot{q}+G^{\prime} \dot{q}+K^{\prime} q=0
$$

As an alternative to the use of Eq. (77), with advantage taken of Eq. (79), one can introduce a real transformation of Eq. (80) which also facilitates truncation and computation.

The eigenvalues of the differential operator in Eq. (89) are in the stable case of interest purely imaginary, and may be designated

$$
\pm i p_{r}, r=1, \cdots, 6 n, i \triangleq(-1)^{1 / 2}
$$

and the corresponding complex eigenvectors may be designated $\psi^{r}+i \gamma^{r}$, with $\psi^{r}$ and $\gamma^{r}$ representing real $(6 n \times 1)$ matrices. If now $\psi$ is constructed as the $(6 n \times 6 n)$ matrix whose columns are $\psi^{1}, \cdots, \psi^{6 n}$, and $\gamma$ is constructed as the $(6 n \times 6 n)$ matrix whose columns are $\gamma^{1}, \cdots, \gamma^{6 n}$, and $p$ is assembled as the $(6 n \times 6 n)$ diagonal matrix with elements $p_{1}, \cdots, p_{6 n}$, then the transformation

$$
q=\psi z+\gamma p^{-1} \dot{z}
$$

may be shown to reduce Eq. (89) to the uncoupled form

$$
\ddot{z}+p^{2} z=0
$$

where $z$ is a $(6 n \times 1)$ real matrix of new coordinates. To verify this transformation, one can recast $\mathrm{Eq}$. (89) as a state equation (see Eq. 80), and introduce the transformation

$$
Q=J Z
$$

4This result was obtained in Ref, 8 , but by an argument which relied incorrectly upon the commutativity of the truncation and inversion operations on $\Phi$. The error was noted by Dr. W. Hooker of Lockheed Palo Alto Research Laboratories, and he provided the alternative argument, noting its approximate nature. where in partitioned form

$$
J \triangleq\left[\begin{array}{c:c}
\psi & \gamma \\
\hdashline-\gamma p & \psi p
\end{array}\right]
$$

By actually solving the homogeneous form of Eq. (80) in terms of $Z$ explicitly, (see Ref. 8, pp. 47-51), one finds that $Z$ and $Z$ are related by

$$
\dot{Z}=\left[\begin{array}{c:c}
0 & p \\
\hdashline-p & 0
\end{array}\right] Z
$$

so that if the partitioned upper half of $Z$ is called $z$, one may write

$$
Z=\left[\begin{array}{c}
z \\
\hdashline-\bar{p} \bar{z}
\end{array}\right]
$$

Then the partitioned bottom half of Eq. (94) confirms Eq. (91). Furthermore, by comparing Eq. (94) with the result of substituting Eq. (90) into the homogeneous form of Eq. (80) and premultiplying by $J^{-1}$, one obtains

$$
J^{-1} B J=\left[\begin{array}{c:c}
0 & p \\
\hdashline-p & 0
\end{array}\right]
$$

Although the homogeneous Eq. (89) is transformed by Eq. (90) into the uncoupled second-order form of Eq. (91) there exists no transformation which uncouples the corresponding second-order inhomogeneous equations. Since the latter is of paramount interest, one must in simulation deal with first-order equations. Equation (91) serves to guide the selection of those $N$ modes of dynamic response deemed significant for the purposes at hand, permitting the construction of the $(6 n \times N)$ matrices $\bar{\psi}$ and $\bar{\gamma}$ and the $(N \times N)$ matrix $p$ from selected elements of $\psi, \gamma$, and $p$. These truncated (barred) matrices are then combined according to the pattern of Eq. (93) to establish a matrix $J$ and the substitution

$$
Q=\bar{J} \bar{Z}
$$

into Eq. (80) is imposed to obtain its approximate solution. Here $\bar{Z}$ is a $(2 N \times 1)$ matrix, so that $\mathrm{Eq}$. (97) provides constraints upon the $(12 n \times 1)$ matrix $Q$, precluding the representation of the general solution of Eq. (80) in terms of the variables in $\bar{Z}$. If the restrictions imposed by Eq. (97) are acceptable, and its substitution into Eq. (80) is followed by premultiplication by the pseudoinverse $\bar{J}^{+}$, the result is

$$
\dot{\bar{Z}}=\bar{J}^{\dagger} B \bar{J} \bar{Z}+\bar{J}^{\dagger} L
$$


Equation (96) suggests, but does not obviously imply, that

$$
\bar{J}+B \bar{J}=\left[\begin{array}{c:c}
0 & p \\
\hdashline-\bar{p} & 0
\end{array}\right]
$$

This relationship is proven in the Appendix.

The pseudoinverse $\bar{J}^{\dagger}$ in Eq. (98) can then be expressed in the manner of Eq. (82) to obtain a differential equation suitable for simulation in the form

$$
\dot{\bar{Z}}=\left[\begin{array}{c:c}
0 & \bar{p} \\
\hdashline-\bar{p} & 0
\end{array}\right] \bar{Z}+\left(\bar{J}^{T} \bar{J}\right)^{-1} \bar{J}^{T} L
$$

In comparing the alternative truncated modal equations (Eqs. 77, 88, and 100) for their relative advantages and disadvantages in simulation, one should note that only the last of these avoids complex variables, while only the first preserves the rigor associated with accomplishing the coordinate truncation only after transformation to uncoupled equations has been accomplished. The first method requires, in general, the computation of the adjoint eigenvectors, but no matrix inversion. The last approach is restricted to the special case of Eq. (64) for which $D^{\prime}=A^{\prime}=0$, so that in the eigenvalue analysis Eq. (89) replaces the homogeneous counterpart to Eq. (64). This restriction also permits the use of Eq. (79) to simplify the task of obtaining Eq. (77) and simplifies eigenvalue/eigenvector computations. When, in addition, the matrices $M^{\prime}$ and $G^{\prime}$ in Eq. (89) are banded along the main diagonal, special computational algorithms (Ref. 21) may be employed to further reduce analysis time, but since this form can be achieved only by ignoring for eigenvalue analysis the contributions of $M^{*}$ and $M^{c}$ to $M^{\prime}$ in Eq. (65) and the similar term in $G^{\prime}$, advantage can be taken of such algorithms only in special applications.

\section{Perspective}

The end result of this paper is a system of differential equations (Eq. 64 or its constituent parts, Eqs. 62, 53, and 46) which characterize the vibratory deformations of a flexible structure attached to a rotating base, together with several alternative forms of the transformed and truncated modal equations suitable for simulation (Eqs. 77, 88, and 100). Even after transformation these equations are an incomplete set, requiring augmentation by additional dynamical, kinematical, and control law equations in the case of spacecraft application.

References 7 and 8 treat the total question of the hybrid coordinate approach to the simulation of spacecraft with elastic appendages, and in Refs. 13-15 the practical utility of this method in application to spacecraft of realistic complexity is demonstrated. This method requires as input a system of appendage equations with an appropriate transformation to modal coordinates. It is the purpose of the present paper to provide that input, for a mathematical model of a flexible appendage more general than any heretofore considered-namely a finite element, distributed mass model. This representation of a flexible appendage is shown to possess an important new advantage over the nodal body approach, in addition to those previously noted (Ref. 22), in that for a vehicle with constant nominal spin the matrix $A^{\prime}$ in Eq. (64) disappears for the finite element model and survives for an arbitrary collection of nodal bodies. Since the elimination of $A^{\prime}$ is an important step in reducing Eq. (64) to one of several forms admitting more convenient modal coordinate transformation than is possible in the general case, this is a potentially important advantage for distributed mass, finite element analysis. 


\section{Appendix}

\section{Proof of Eq. (99)}

To prove Eq. (99), note that Eq. (80) admits the solution

$$
\left[\begin{array}{c}
q \\
\dot{q}
\end{array}\right]=\Phi^{r} e^{\lambda_{r} t}
$$

where $\lambda_{r}$ is an eigenvalue and $\Phi^{r}$ the corresponding eigenvector of $B$, and infer the form

$$
\Phi^{r}=\left[\begin{array}{c}
\phi^{r} \\
-\phi^{r} \lambda_{r}^{-}
\end{array}\right]
$$

so that $\Phi$ may be written in partitioned form as

$$
\Phi=\left[\begin{array}{c:c}
\phi & \phi^{*} \\
\hdashline \phi \bar{\lambda} & \phi^{*} \lambda^{*}
\end{array}\right]
$$

( ${ }^{*}$ representing complex conjugate). The substitutions

$$
\phi=\psi+i \gamma, \quad \lambda=i p
$$

provide

$$
\Phi=\left[\begin{array}{c:c}
\psi & \psi \\
\hdashline-\gamma p & -\gamma p
\end{array}\right]+i\left[\begin{array}{c:c}
\gamma & -\gamma \\
\hdashline \psi p & =\psi p
\end{array}\right]
$$

which when substituted with

$$
\Lambda=i\left[\begin{array}{c:c}
p & 0 \\
\hdashline 0 & -p
\end{array}\right]
$$

into Eq. (84) provides from its real and imaginary parts the two equations

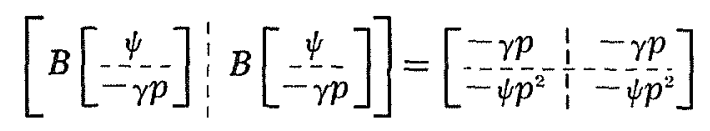

and

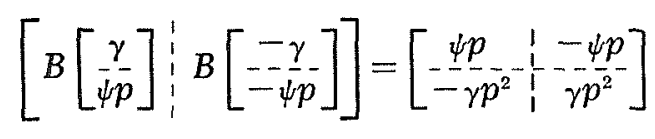

With the additional partitioning

$$
\psi=[\bar{\psi}: \overline{\bar{\psi}}] \quad \gamma=[\bar{\gamma}: \overline{\bar{\gamma}}] \quad p=\left[\begin{array}{c:c}
\bar{p} & 0 \\
\hdashline \overline{0} & \overline{\bar{p}}
\end{array}\right]
$$

one can extract from Eqs. (A-7) and (A-8) the equations

$$
B\left[\begin{array}{c}
\bar{\psi} \\
-\overline{\gamma \bar{p}}
\end{array}\right]=\left[\begin{array}{c}
-\overline{\gamma p} \\
-\bar{\psi} \overline{\psi p}
\end{array}\right]
$$

and

$$
B\left[\begin{array}{c}
\bar{\gamma} \\
\bar{\psi} \overline{\psi p}
\end{array}\right]=\left[\begin{array}{c}
\overline{\psi p} \\
-\overline{\gamma \bar{p}^{2}}
\end{array}\right]
$$

Substitution of the definition of $B$ following Eq. (80) and expansion of Eqs. (A-10) and (A-11) lead to the two independent equations

$$
M^{\prime-1} K^{\prime} \bar{\psi}-M^{\prime-1} G^{\prime} \bar{\gamma} \bar{p}=\bar{\psi} \bar{p}^{2}
$$

and

$$
M^{\prime-1} K^{\prime} \bar{\gamma}+M^{\prime-1} G^{\prime} \bar{\psi} \bar{p}=\bar{\gamma} \bar{p}^{2}
$$

as well as a group of identities.

Equation (99) requires the evaluation of

$$
\bar{J}^{+} B \bar{J}=\left(\bar{J}^{T} \bar{J}\right)^{-1} \bar{J}^{T} B \bar{J}
$$

Definitions provide

$$
\begin{aligned}
B \bar{J} & =\left[\begin{array}{c:c}
0 & U \\
\hdashline \bar{M}^{\prime-1} \bar{K}^{\prime} & -\bar{M}^{\prime-1} G^{\prime}
\end{array}\right]\left[\begin{array}{c:c}
\bar{\psi} & \bar{\gamma} \\
\hdashline \overline{\gamma p} & \overline{\psi p}
\end{array}\right] \\
& =\left[\begin{array}{ccc}
-\overline{\gamma p} & \overline{\psi p} \\
\hdashline M^{\prime-1} \bar{K}^{\prime} \bar{\psi}+\bar{M}^{\prime-1} G^{\prime} \overline{\gamma p} & -M^{\prime-1} K^{\prime} \bar{\gamma}-\bar{M}^{\prime-1} G^{\prime} \overline{\psi p}
\end{array}\right]
\end{aligned}
$$

which with Eqs. (A-12) and (A-13) becomes

$$
B J=\left[\begin{array}{c:c}
-\overline{\gamma p} & \overline{\psi p} \\
\hdashline-\bar{\psi} \bar{p}^{2} & -\overline{\gamma p^{2}}
\end{array}\right]=\left[\begin{array}{c:c}
-\bar{\gamma} & \bar{\psi} \\
\hdashline-\overline{\psi p} & -\overline{\gamma p}
\end{array}\right]\left[\begin{array}{c:c}
\bar{p} & 0 \\
\hdashline 0 & \bar{p}
\end{array}\right]
$$

providing

$$
\bar{J}^{T} B \bar{J}=\left[\begin{array}{c:c}
\bar{\psi}^{T} \bar{\gamma}+\bar{\gamma}^{T} \overline{\psi p}^{2} & \bar{\psi}^{T} \bar{\psi}+\bar{\gamma}^{T} \bar{\gamma}^{2} \\
\hdashline \bar{\gamma}^{T} \bar{\gamma}-\bar{\psi}^{T} \bar{\psi} \bar{p}^{2} & \bar{\gamma}^{T} \bar{\psi}-\bar{\psi}^{T} \bar{\gamma} \bar{p}^{2}
\end{array}\right]\left[\begin{array}{c:c}
\bar{p} & 0 \\
\hdashline 0 & \bar{p}
\end{array}\right]
$$


Comparing Eq. (A-17) with

$$
\bar{J}^{T} \bar{J}=\left[\begin{array}{c:c}
\bar{\psi}^{T} \bar{\psi}+\bar{\gamma}^{T} \overline{\gamma p}^{2} & \bar{\psi}^{T} \bar{\gamma}-\bar{\gamma}^{T} \bar{\psi}^{2} \\
\hdashline \bar{\gamma}^{T} \bar{\psi}-\bar{\psi}^{T} \overline{\gamma p^{2}} & \bar{\gamma}^{T} \bar{\gamma}+\bar{\psi}^{T} \bar{\psi} \bar{p}^{2}
\end{array}\right]
$$

indicates that Eq. (A-14) is of the form

$$
\bar{J}^{+} B \bar{J}=\left[\begin{array}{c:c:c}
a & c \\
\hdashline c^{T} & b
\end{array}\right]^{-1}\left[\begin{array}{c:c}
-c & a \\
\hdashline-b & c^{p}
\end{array}\right]\left[\begin{array}{c:c}
\bar{p} & 0 \\
\hdashline 0 & \bar{p}
\end{array}\right]
$$

or, using the formula (Ref. 23) for matrix inversion in terms of partitions,

$$
\bar{J}+B \bar{J}=\left[\begin{array}{c:c}
A & B \\
\hdashline C & D
\end{array}\right]\left[\begin{array}{c:c}
\bar{p} & 0 \\
\hdashline 0 & \bar{p}
\end{array}\right]
$$

where

$$
\begin{aligned}
& A \triangleq-\left(a-c b^{-1} c^{T}\right)^{-1} c+a^{-1} c\left(b-c^{T} a^{-1} c\right)^{-1} b \\
& B \triangleq\left(a-c b^{-1} c^{T}\right)^{-1} a-a^{-1} c\left(b-c^{T} a^{-1} c\right)^{-1} c^{T} \\
& C \triangleq b^{-1} c^{T}\left(a-c b^{-1} c^{T}\right)^{-1} c-\left(b-c^{T} a^{-1} c\right)^{-1} b \\
& D \triangleq-b^{-1} c^{T}\left(a-c b^{-1} c^{T}\right)^{-1} a+\left(b-c^{T} a^{-1} c\right)^{-1} c^{T}
\end{aligned}
$$

Note that

$$
A+B a^{-1} c=a^{-1} c
$$

$$
\begin{aligned}
& A b^{-1} c^{T}+B=U \\
& C+D a^{-1} c=-U \\
& C b^{-1} c^{T}+D=-b^{-1} c^{T}
\end{aligned}
$$

Equations (A-21) and (A-22) may be combined as

$$
\left(A+B a^{-1} c\right) b^{-1} c^{T}-\left(A b^{-1} c^{T}+B\right)=a^{-1} c b^{-1} c^{T}-U
$$

which is equivalent to

$$
B\left(a^{-1} c b^{-1} c^{T}-U\right)=\left(a^{-1} c b^{-1} c^{T}-U\right)
$$

implying $B=U$. Thus from Eq. (A-21) the matrix $A=0$. Similarly, Eqs. (A-23) and (A-2A) combined as

$$
\left(C b^{-1} c^{T}+D\right) a^{-1} c-\left(C+D a^{-1} c\right)=-b^{-1} c^{T} a^{-1} c+U
$$

provide

$$
C=-U \quad D=0
$$

Thus Eq. (A-20) becomes

$$
\bar{J}^{+} B \bar{J}=\left[\begin{array}{c:c}
0 & U \\
\hdashline-U & 0
\end{array}\right]\left[\begin{array}{c:c}
\bar{p} & 0 \\
\hdashline 0 & \bar{p}
\end{array}\right]=\left[\begin{array}{c:c}
0 & \bar{p} \\
\hdashline-\bar{p} & 0
\end{array}\right]
$$

proving Eq. (99). 


\section{Nomenclature}

English Symbols

A inertial acceleration of point $p$

$\bar{A} \quad(3 \times 1)$ matrix of $\mathbf{A}$ in basis $\{\mathbf{e}\}$

$\mathbf{A}^{j}$ inertial acceleration of node $j$

A $(12 n \times 12 n)$ coefficient matrix in Eq.(71)

a reference frame established by $Q$ and $a_{1}, a_{2}, a_{3}$

$\mathbf{a}_{1}, \mathbf{a}_{2}, \mathbf{a}_{3}$ dextral, orthogonal set of unit vectors fixed in $a$

\{a\} (3×1) vector array with elements $\mathbf{a}_{1}, \mathbf{a}_{2}, \mathbf{a}_{3}$

$B \quad(12 n \times 12 n)$ coefficient matrix in Eq. (80)

B $(12 n \times 12 n)$ coefficient matrix in Eq. (71)

$b$ reference frame established by base body

$\mathbf{b}_{1}, \mathbf{b}_{2}, \mathbf{b}_{3}$ dextral, orthogonal set of unit vectors fixed in $b$

C $(3 \times 3)$ variable direction cosine matrix; $\{\mathbf{a}\}=C\{\mathbf{b}\}$

$\bar{C} \quad(3 \times 3)$ constant direction cosine matrix; $\{\mathbf{e}\}=\bar{C}\{\mathbf{a}\}$

$C M$ vehicle mass center

$\bar{C}^{s} \quad \bar{C}$ for element $s$, so $\left\{\mathbf{e}^{s}\right\}=C^{s}\{\mathbf{a}\}$

$\mathrm{CM}^{s^{\prime}}$ sth element mass center when appendage undeformed

$C M^{s} \quad s$ th element mass center when appendage in steady state

c $(3 \times 1)$ matrix of $\mathrm{c}$ in basis $\{\mathrm{a}\}$

c vector from $C M$ to point $O$

$c^{\prime}$ vector from $C M$ to point $O^{\prime}$

$c_{i j}$ element of $C, i, j=1,2,3$

$D$ displacement coefficient matrix in $\epsilon=D \bar{w}$ (Eq. (12))

dv differential element of volume

$E$ Young's modulus

$\mathcal{E}$ number of finite elements

$\delta_{j}$ set of indices of finite elements in contact with node $j$ $e \quad(3 \times 1)$ matrix of $e$ in basis $\{a\}$

e contribution to c not attributable to appendage deformation, Eq. (43)

$\mathbf{e}_{1}, \mathbf{e}_{2}, \mathbf{e}_{3}$ dextral, orthogonal set of unit vectors fixed in generic element under steady-state deformation

\{e\} $(3 \times 1)$ vector array with elements, $\mathfrak{e}_{1}, \mathfrak{e}_{2}, \mathbb{e}_{3}$

$F \quad(6 n \times 6 n)$ matrix relating $y$ to $\Gamma, \mathbb{E q}$. (9)

$\mathbf{F}^{j}$ resultant force on $j$ th nodal body

$F^{j} \quad(3 \times 1)$ matrix of $F^{j}$ in basis $\{a\}$

$\bar{F}^{j s} \quad(3 \times 1)$ matrix in basis $\{\mathbf{e}\}$ of force applied by body $j$ to element $s$

$\mathbf{F}^{s j}$ force applied by finite element $s$ to nodal body $i$

$\bar{F}^{s j} \quad(3 \times 1)$ matrix of $F^{s j}$ in basis $\{\mathrm{e}\}$

$f^{j}$ resultant for nodal body $j$ of forces external to the system

$f^{3} \quad(3 \times 1)$ matrix of $f^{j}$ in basis $\{a\}$

$f, g$ arbitrary reference frames

$\bar{G}(\xi, \eta, \zeta) \quad(3 \times 1)$ matrix function of ellement body forces in basis $\{\mathbf{e}\}$

$G^{\prime} \quad(6 n \times 6 n)$ gyroscopic coupling matrix in Eq. (64)

$\bar{g}$ element gyroscopic coupling matrix $(6 n \times 6 n)$

$\mathbf{H}^{j} \quad$ angular momentum of nodal body $j$ for its mass center

$\|^{j} \quad$ inertia dyadic of nodal body $j$ for its mass center

$I^{j} \quad(3 \times 3)$ inertia matrix for $\mathbb{1}^{j}$ in basis $\left\{\mathrm{n}^{j}\right\}$

$I_{\alpha \gamma}$ element of $I^{j} ; \alpha, \gamma=1,2,3$

\& inertially fixed point

$i(-1)^{1 / 2}$

\{i\} $(3 \times 1)$ vector array of inertially fixed, dextral, orthogonal unit vectors $i_{1}, i_{2}, i_{3}$

$J, \bar{J}$ transformation matrix in Eq. (92), in $(12 n \times 12 n)$ and truncated $(2 N \times 2 N)$ forms 


\section{Nomenclature (contd)}

$\mathbb{K}^{\prime} \quad(6 n \times 6 n)$ appendage stiffness matrix (Eq. 64)

$\bar{k}, k \quad(6 n \times 6 \bigcap)$ finite element structural stiffness matrix, for vector bases $\{\mathbf{e}\}$ and $\{\mathbf{a}\}$ respectively

$\bar{k}_{0} \quad(6 n \times 6 n)$ stiffness matrix for unloaded element for basis $\{\mathbf{e}\}$

$\bar{k}_{\Delta} \quad(6 n \times 6 n)$ preload (geometric) stiffness matrix for element, basis $\{\mathbf{e}\}$

$\bar{L}$ generic $L^{s}$

$\bar{L}^{s} \quad(6 n \times 1)$ matrix of forces and torques on the nodes of element $s$, Eq. (19)

$L^{\prime} \quad(6 n \times 1)$ matrix in Eq. (64)

$\& \quad(12 n \times 1)$ matrix in Eq. (71)

$M^{\prime} \quad(6 n \times 6 n)$ generalized inertia matrix, Eq. (64)

$M, M^{c}, M^{*} \quad$ constituents of $M^{\prime}$, Eq. (65)

$M_{i j}^{c}$ element of $M^{c} ; i, j=1, \cdots, 6 n$

$m$ vehicle mass

$m_{s} \quad$ mass of finite element $s$

$\bar{m}, m \quad(6 n \times 6 n)$ consistent mass matrix for finite element, bases $\{\mathbf{e}\}$ and $\{\mathbf{a}\}$ respectively, Eqs. (35), (40)

$m_{j}, m^{j}$ mass of $j$ th nodal body, and mass matrix $m^{j}=m_{j} U$

$N$ number of modal coordinates after truncation

$n_{3}, n$ number of nodes for finite element $s$, and generic $n_{s}$

$n$ number of nodes in appendage

$\mathrm{n}_{1}^{j}, \mathrm{~m}_{2}^{j}, \mathrm{n}_{3}^{j}$ dextral, orthogonal set of unit vectors fixed in nodal body $j$ (superscript omitted for generic symbol)

\{n\} (3× $)$ vector array with elements $\mathbf{n}_{1}, \mathbf{n}_{2}, \mathbf{n}_{3}$

$O$ point fixed in $b$, and vehicle $C M$ for steadystate deformation

$O^{\prime}$ point fixed in $b$, and vehicle $C M$ for appendage undeformed
$P \quad(3 \times 6, n)$ matrix relating $\bar{w}$ to $\Gamma$, Eq. $(5)$

$p \quad(6 n \times 6 n)$ diagonal matrix with nonzero elements $p_{1}, \cdots, p_{6 n}$

$\bar{p} \quad(N \times N)$ matrix truncation of $p$

$p_{r}$ natural frequency from Eq. (89)

$Q \quad(12 n \times 1)$ state matrix, Eq. (71)

Q point common to $a$ and $b$

$q \quad(6 n \times 1)$ matrix of variational deformation variables, Eq. (63)

$\mathbf{R}, \boldsymbol{R}$ vector from $O$ to $Q$, and $(3 \times 1)$ matrix in basis $\{\mathbf{a}\}$

$\mathbf{R}^{\prime}, R^{\prime}$ vector from $O^{\prime}$ to 2 , and $(3 \times 1)$ matrix in basis $\{a\}$

$\mathbf{R}_{c}^{s}, R_{c}^{s} \quad$ vector from $Q$ to $C M^{s}$, and $(3 \times 1)$ matrix in basis $\{a\}$

$\mathbf{R}_{c}, \boldsymbol{R}_{c} \quad$ generic $\mathbf{R}_{c}^{s}, R_{c}^{s}$

$\mathbf{R}_{c}^{s \prime}$ vector from $\&$ to $C M^{s^{\prime}}$

$\mathbf{r}^{j}, r^{j}$ vector from $@$ to steady-state node $j$, and $(3 \times 1)$ matrix in basis $\{\mathbf{a}\}$

$\mathbf{r}^{j \prime} \quad$ vector from $Q$ to node $j$ of undeformed structure

$S(6 \times 6)$ coefficient matrix in stress-strain equation (15)

$\mathbb{T}^{j}, T^{j}$ torque on nodal body $j$, vector and $(3 \times 1)$ matrix in basis $\{\mathbf{a}\}$, respectively

$\mathbf{T}^{s j}, \bar{T}^{s j} \quad$ torque on nodal body $j$ applied by element $s$, vector and $(3 \times 1)$ matrix in basis $\{\mathbf{e}\}$, respectively

$\mathbf{t}^{j}, t^{j} \quad$ external torque on nodal body $i$, vector and $(3 \times 1)$ matrix in basis $\{a\}$, respectively

$U \quad(3 \times 3)$ unit matrix

$Q^{*} \quad$ virtual strain energy

$\mathbf{u}^{j}, u^{j}$ displacement of node $j$ due to variations from steady-state deformation (i.e., variational translational nodal deformation), and $(3 \times 1)$ matrix of $\mathbf{u}^{j}$ in basis $\{a\}$

$\mathbf{u}^{j \prime}$ displacement of node $j$ due to steady-state deformation 


\section{Nomenclafure (contd)}

W $(3 \times 6 N)$ matrix relating $\bar{w}$ to $\bar{y}$, Eq. (11)

(2) $)^{* *}$ virtual work

$w \quad(3 \times 1)$ matrix of $w$ in basis $\{\mathbf{a}\}$

$\mathbf{w}^{\prime}$ displacement of field point of finite element due to steady-state deformation

$\mathbf{w}^{s}$ displacement of field point of finite element $s$ due to variations from steady-state deformation (i.e., variational element deformation)

$\bar{w}^{s} \quad(3 \times 1)$ matrix of $\mathbf{w}^{s}$ in basis $\{\mathbf{e}\}$

$\mathrm{w}, \bar{w}$ generic for $\mathbf{w}^{s}$ and $\bar{w}^{s}$

$X, X$ vector from $\theta$ to $C M$, and $(3 \times 1)$ matrix in basis $\{\mathbf{i}\}$

$x, y$ arbitrary $(3 \times 1)$ matrices

$Y, \bar{Y} \quad(12 n \times 1)$ transformed state variable matrix, Eq. (74) and $(2 N \times 1)$ truncated form

$\bar{y}^{s}, \bar{y} \quad\left(6 n_{s} \times 1\right)$ matrix of deformational nodal displacements for finite element $s$, and generic form

$Z, \bar{Z} \quad(12 n \times 1)$ transformed state variable matrix, Eq. $(92)$ and $(2 N \times 1)$ truncated form

$z(6 n \times 1)$ matrix of transformed variables, Eq. (91)

\section{Greek Symbols}

$\bar{\alpha}$ coefficient of thermal expansion

$\beta_{\alpha}^{j} \quad$ rotation of nodal body $j$ for axis $\mathbf{a}_{\alpha}$ due to variations of deformation from steady state (i.e., variational rotational nodal deformation) $(\alpha=1,2,3)$

$\beta^{j}, \beta^{j} \quad \beta_{1}^{j} \mathbf{a}_{1}+\beta_{2}^{j} \mathbf{a}_{2}+\beta_{3}^{j} \mathbf{a}_{3}$, and $(3 \times 1)$ matrix in basis $\{a\}$

$\beta_{\alpha}^{j \prime}$ rotation of nodal body $j$ for axis $\alpha$ due to steady-state deformation $(\alpha=1,2,3)$

T $(6 n \times 1)$ matrix in Eq. (5)

$\gamma \quad(6 n \times 6 n)$ matrix with columns $\gamma^{1}, \cdots, \gamma^{6 n}$ the real parts of eigenvectors of Eq. (89) $\bar{\epsilon} \quad(6 \times 1)$ strain matrix due to variations from steady-state deformation, Eq. (12)

$\bar{\epsilon}^{\prime} \quad$ steady-state strain matrix $(6 \times 1)$

$\bar{\epsilon}_{\tau} \quad(6 \times 1)$ strain matrix due to deviations from steady-state temperature

$\epsilon_{\alpha \gamma \theta}$ epsilon symbol of tensor analysis

$\bar{\epsilon}_{\alpha \gamma} \quad$ strain element, basis $\{\mathbf{e}\}$

$\xi, \eta, \zeta$ Cartesian coordinates corresponding to $\mathbf{e}_{1}, \mathbf{e}_{2}, \mathbf{e}_{3}$ and origin fixed in element under steady-state deformation

$\Theta \quad(3 \times 3)$ direction cosine matrix in $\{\mathbf{b}\}=\Theta\{\mathbf{i}\}$

$\bar{\kappa}^{s}, \bar{\kappa} \quad(6 n \times 6 n)$ centripetal stiffness matrix for finite elements, and generic $\bar{\kappa}^{s}$

$\Lambda, \bar{\Lambda} \quad(12 n \times 12 n)$ diagonal matrix of eigenvalues of $B$, and truncated $(2 N \times 2 N)$ form

$\mu \quad$ mass density of finite element

$v$ Poisson's ratio

$\rho^{s^{\prime}}$ position vector to field point of undeformed element $s$ from $C M^{s^{\prime}}$

$\boldsymbol{\rho}^{\delta}, \bar{\rho}^{s}$ position vector and $(3 \times \mathbb{1})$ matrix in $\{\mathbf{e}\}$ basis to field point of element $s$ in steady state from $C M^{s}$

$\rho, \bar{\rho}$ generic $\rho^{s}$ and $\bar{\rho}^{s}$

$\bar{\sigma}(6 \times 1)$ stress matrix, Eqs. (16), (17)

$\bar{\sigma}^{\prime} \quad$ steady-state stress (e.g., due to spin)

$\bar{\sigma}_{\alpha \gamma}$ stress due to deviation from steady-state deformation, $\{\mathbf{e}\}$ basis

$\bar{\sigma}_{\tau} \quad(6 \times 1)$ stress matrix accommodating thermal strains

$\tau$ variation from steady-state temperature

$\Phi(12 n \times 12 n)$ transformation matrix of eigenvectors, Eq. (74)

$\Phi^{\prime} \quad(12 n \times 12 n)$ matrix of adjoint eigenvectors of Eq. (71), see Eq. (72)

$\psi \quad(6 n \times 6 n)$ real matrix with columns $\psi^{1}, \cdots, \psi^{6 n}$, the imaginary parts of eigenvectors of Eq. (89) 


\section{Nomenclature (contd)}

\footnotetext{
$\Omega$ nominal value of $\omega^{a}$, with elements $\Omega_{1}, \Omega_{2}, \Omega_{3}$

$\omega^{j} \quad$ inertial angular velocity of nodal body $j$

$\omega^{a}{ }_{,} \omega^{a}$ inertial angular velocity vector and $(3 \times 1)$ matrix in $\{a\}$ basis of frame a

$\omega^{f g}$ angular velocity of frame $f$ relative to frame $g$
$\omega, \omega$ inertial angular velocity vector and $(3 \times 1)$ matrix in basis $\{\mathbf{b}\}$ of frame $b$

Operational Symbols

()$^{T}$ indicates matrix transposition $(\tilde{)}$ or $\tilde{C})$ indicates formation of $(3 \times 3)$ skewsymmetric matrix from $(3 \times 1)$ matrix, as in Eq. (30)

$\frac{\prime d}{d t}(\mathrm{~V})$ time derivative of arbitrary vector $\mathrm{V}$ in reference frame $f$

(C) time derivative of scalar or matrix

( ) virtual quantity (stress, displacement, etc.); also conjugate (not conjugate transpose)

()$^{\dagger}$ pseudoinverse of rectangular matrix

()$^{-1}$ matrix inverse

$\triangleq \quad$ means equality by definition

Repeated lower case Greek indices indicate summation over range $1,2,3$. 


\section{References}

1. Hooker, W. W., and Margulies, G., "The Dynamical Attitude Equations for an n-Body Satellite," J. Astronaut. Sci., Vol. 12, pp. 123-128, 1965.

2. Roberson, R. E., and Wittenburg, J., "A Dynamical Formalism for an Arbitrary Number of Interconnected Rigid Bodies, with Reference to the Problem of Satellite Attitude Control," Proc. 3rd Int. Congress of Automat. Cont. (London, 1966), Butterworth and Co., Ltd., London, 1967.

3. Ashley, H., "Observations on the Dynamic Behavior of Large, Flexible Bodies in Orbit," AIAA J., Vol. 5, pp. 460 469, 1967.

4. Likins, P. W., "Modal Method for Analysis of Free Rotations of Spacecraft," AIAA J., Vol. 5, pp. 1304-1308, 1967.

5. Gevarter, W. B., "Basic Relations for Control of Flexible Vehicles," AIAA J., Vol. 8, pp. 666-672, 1970.

6. Likins, P. W., and Wirsching, P. H., "Use of Synthetic Modes in Hybrid Coordinate Dynamic Analysis," AIAA J., Vol. 6, pp. 1867-1872, 1968.

7. Likins, P. W., and Gale, A. H., "Analysis of Interactions Between Attitude Control Systems and Flexible Appendages," Proc. 19th Int. Astronaut. Congress, Vol. 2, pp. 67-90, Pergamon Press, 1970.

8. Likins, P. W., Dynamics and Control of Flexible Space Vehicles, Technical Report 32-1329, Rev. 1. Jet Propulsion Laboratory, Pasadena, Calif., Jan. 15, 1970.

9. Meirovitch, L., and Nelson, H. D., "On the High-Spin Motion of a Satellite Containing Elastic Parts,” J. Spacecraft Rockets, Vol. 3, pp. 1597-1602, 1966.

10. Vigneron, F., "Stability of a Freely Spinning Satellite of Crossed Dipole Configuration," C.A.S.I. Trans., Vol. 3, No. 1, pp. 8-19, Mar. 1970.

11. Rakowsky, J. F., and Renard, M. L., "A Study of the Nutational Behavior of a Flexible, Spinning Satellite Using Natural Frequencies and Modes of the Rotating Structure," AIAA Paper 70-1046, presented at AAS/AIAA Astrodynamics Conference, Santa Barbara, Calif., Aug. 19-21, 1970.

12. Grote, D. B., McMunn, J. C., and Gluck, R., "Equations of Motion of Flexible Spacecraft," AIAA Paper 70-19, presented at AIAA 8th Aerospace Sciences Meeting, New York, Jan. 19-21, 1970.

13. Likins, P. W., and Fleischer, G. E., "Results of Flexible Spacecraft Attitude Control Studies Utilizing Hybrid Coordinates," J. Spacecraft Rockets, Vol. 8, pp. 264-272, 1971.

14. Marsh, E. L., "The Attitude Control of a Flexible, Solar Electric Spacecraft," presented at AIAA Electric Propulsion Conference, Stanford University, Stanford, Calif., Aug. 1970.

15. Gale, A. H., and Likins, P. W., "Influence of Flexible Appendages on Dual-Spin Spacecraft Dynamics and Control," J. Spacecraft Rockets, Vol. 7, pp. 1049-1056, 1970.

16. Wilkinson, J. H., The Algebraic Eigenvalue Problem, p. 4, Clarendon Press, Oxford, 1965. 


\section{References (contd)}

17. Greville, T. N. E., "The Pseudoinverse of a Rectangular or Singular Matrix and Its Application to the Solution of Systems of Equations," SIAM Rev., Vol. 1, pp. $38-43,1959$.

18. Foss, K. A., "Co-ordinates Which Uncouple the Equations of Motion of Damped Linear Dynamic Systems," J. Appl. Mech., Vol. 25, pp. 361-364, 1958.

19. Caughey, T. K., "Classical Normal Modes in Damped, Linear, Dynamic Systems," J. Appl. Mech., Vol. 27, pp. 269-271, 1960.

20. Whittaker, E. T., A Treatise on the Analytical Dynamics of Particles and Rigid Bodies, 4th ed. Cambridge University Press, 1937, pp. $427 \mathrm{ff}$.

21. Gupta, K. K., "Eigenvalue of $\left(E^{*}-w F\right) y=0$ with Positive Definite Band Symmetric $F$ and Band Hermitian $E^{*}$ and Its Application to Free Vibration Analysis of Rotating Structures." SIAM 1970 Fall Meeting, Boston, Mass., Oct. 12-14, 1970.

22. Archer, J. S., "Consistent Mass Matrix for Distributed Systems," Proc. A.S.C.E., 89, ST 4, p. 161, 1963.

23. Korn, G. A., and Korn, T. M., Mathematical Handbook for Scientists and Engineers, p. 640. McGraw-Hill Book Co., Inc., New York, 1961. 